ACCEPTED FOR PUBLICATION IN THE ASTROPHYSICAL JOURNAL

Preprint typeset using LATEX style emulateapj v. 04/17/13

\title{
MEASURING THE PROGENITOR MASSES AND DENSE CIRCUMSTELLAR MATERIAL OF TYPE II SUPERNOVAE
}

\author{
VikTORIYA Morozova ${ }^{1}$, Anthony L. Piro ${ }^{2}$, AND Stefano VALEnTI ${ }^{3}$ \\ Accepted for publication in The Astrophysical Journal
}

\begin{abstract}
Recent modeling of hydrogen-rich Type II supernova (SN II) light curves suggests the presence of dense circumstellar material (CSM) surrounding the exploding progenitor stars. This has important implications for the activity and structure of massive stars near the end of their lives. Since previous work focused on just a few events, here we expand to a larger sample of twenty well-observed SNe II. For each event we are able to constrain the progenitor zero-age main-sequence (ZAMS) mass, explosion energy, and the mass and radial extent of dense CSM. We then study the distribution of each of these properties across the full sample of SNe. The inferred ZAMS masses are found to be largely consistent with a Salpeter distribution with minimum and maximum masses of 10.4 and $22.9 M_{\odot}$, respectively. We also compare the individual ZAMS masses we measure with specific SNe II that have pre-explosion imaging to check their consistency. Our masses are generally comparable to or larger than the pre-explosion imaging masses, potentially helping ease the red supergiant problem. The explosion energies vary from $(0.1-1.3) \times 10^{51} \mathrm{erg}$, and for $\sim 70 \%$ of the SNe we obtain CSM masses in the range between $0.18-0.83 M_{\odot}$. We see a potential correlation between the CSM mass and explosion energy, which suggests that pre-explosion activity has a strong impact on the structure of the star. This may be important to take into account in future studies of the ability of the neutrino mechanism to explode stars. We also see a possible correlation between the CSM's radial extent and ZAMS mass, which could be related to the time with respect to explosion when the CSM is first generated.
\end{abstract}

Keywords: hydrodynamics — radiative transfer — supernovae: general

\section{INTRODUCTION}

A longstanding problem in the study of explosive transient events is connecting classes of supernovae ( $\mathrm{SNe})$ to the specific progenitors that generate them. In this sense, the hydrogen-rich Type II SNe should seemingly be the most straightforward for making this connection. With the exception of the rarer Type IIb and 1987A-like events, preexplosion imaging obtained with the Hubble Space Telescope exclusively identifies red supergiants (RSGs) as their progenitors (Li et al. 2006; Smartt et al. 2009; Van Dyk et al. 2012). These are expected to largely evolve as single stars, so that their models can be generated without many of the complications present for other stellar calculations (such as binarity, a high spin, etc). This in turn should simplify explosion and light curve calculations, which find that a RSG naturally produces the plateau-shaped light curve of SNe II with the recombination of many solar masses of hydrogen-rich material with a RSG-like radius (Smith et al. 2011; Utrobin \& Chugai 2013; Dessart et al. 2013; González-Gaitán et al. 2015; Rubin \& Gal-Yam 2016; Renzo et al. 2017).

Nevertheless, there remain many outstanding questions about how Type II progenitors connect to their light curves. Chief among these is the relationship between the Type IIP (plateau) and Type IIL (linear) subclasses, which is based on the shape of their light curves during the first few weeks (Barbon et al. 1979). There has been a long debate on whether there is a physical variable that smoothly transitions between Type IIP and IIL or whether there is a specific mechanism that creates this dichotomy more abruptly. There have been some

\footnotetext{
${ }^{1}$ Department of Astrophysical Sciences, Princeton University, Princeton, NJ 08544, USA; vsg@ astro.princeton.edu

2 The Observatories of the Carnegie Institution for Science, 813 Santa Barbara St., Pasadena, CA 91101

${ }^{3}$ Department of Physics, University of California, Davis, CA 95616
}

claims of distinct populations (Arcavi et al. 2012; Faran et al. $2014 b, a)$, but support for the more continuous case has increased as larger compilations by Anderson et al. (2014) and Sanders et al. (2015) showed a more continuous range of early light-curve slopes. An important breakthrough came when Valenti et al. (2015) demonstrated that if one simply follows an SN IIL long enough, its light curve will drop at $\sim 100$ days, just like a normal SN IIP (previous SNe IIL studies rarely followed the light curve beyond $\sim 80$ days from discovery; see also Anderson et al. 2014). This suggests that Type IIL and Type IIP SNe may have a similar amount of hydrogen present for the main bulk of their envelopes, and whatever is creating the Type IIL distinction may be contributing something above a fairly normal underlying RSG.

Motivated by these issues, Morozova et al. (2017) recently numerically modeled three Type II SNe with extensive photometry over many wavebands by using RSG models with dense CSM stitched on top of them. This work was different from previous theoretical studies (e.g., Moriya et al. 2011) in that the CSM was generally more massive and compact (only extending a few stellar radii above the RSG). The two important conclusions from this work were that (i) this dense CSM could naturally explain the photometric differences between the Type IIP and IIL, and (ii) even the seemingly more normal Type IIP SNe need dense CSM to accurately model their light curves. It is still unclear what the full implications of these results are. It seems to indicate that there is increased activity in RSGs during the last months or years of their lives, which may be related to theoretical studies of pre-explosion outbursts (Quataert \& Shiode 2012; Shiode \& Quataert 2014; Quataert et al. 2016; Fuller 2017). Furthermore, observations of SNe II shortly after explosion show narrow lines that indicate a dense wind-like environment (e.g., Yaron et al. 2017), albeit probing more extended and less dense material than the CSM we need for the light curves (Dessart et al. 2017). This 
in turn brings up the question of whether there is a relationship between these two components of the CSM (Moriya et al. 2017).

An important step toward better understanding this dense CSM is mapping out its diversity over a larger sample of $\mathrm{SNe}$ II. With this goal in mind, we extend our previous work on SN II light curve modeling to a set of twenty especially well-observed events. This allows us to measure the mass and extent of the dense CSM, constrain the zero-age mainsequence (ZAMS) masses, and measure the explosion energies. From this we can derive better constraints on just how common this dense CSM is (at least $70 \%$ of our sample), as well as look for correlations between the various properties that may provide clues to the dense CSM's origin. Beyond just the CSM properties, because we are able to constrain the ZAMS masses for a wide sample, this provides important complementary information about SN II progenitors to other studies of RSGs (for example, work on pre-explosion imaging, Smartt 2009; Smartt et al. 2009).

In Section 2, we describe the details of our simulations. The sample of twenty SNe II used for this work are presented in Section 3 along with our modeling strategy and fitting results. In Section 4, we explore what we can learn with our full sample of fits as well as looking for correlations between different properties of the SN progenitors that we measure. In Section 5, we summarize our results and discuss future work.

\section{NUMERICAL SETUP}

As in our previous work (Morozova et al. 2017) we use the non-rotating solar-metallicity RSG models from the stellar evolution code KEPLER (Weaver et al. 1978; Woosley \& Heger 2007, 2015; Sukhbold \& Woosley 2014; Sukhbold et al. 2016). Above these models we add a CSM extending out to a radius $R_{\text {ext }}$ with the density profile of a steady-state wind

$$
\rho(r)=\frac{\dot{M}}{4 \pi r^{2} v_{\text {wind }}}=\frac{K}{r^{2}},
$$

where $\dot{M}$ is the wind mass loss rate and $v_{\text {wind }}$ is the wind velocity. A steady-state wind is widely used in the literature to describe the structure of CSM in the vicinity of RSGs (Chugai et al. 2007; Ofek et al. 2010; Chevalier \& Irwin 2011; Moriya et al. 2011), and we use it as a convenient prescription to explore the diversity of possible CSM properties with just two parameters, $K$ and $R_{\text {ext }}$ (the case of an accelerating wind is considered in Moriya et al. 2017, while for the cases of exponential or power-law density distributions see Nagy \& Vinkó 2016). We assume the temperature and composition of the CSM to be constant and equal to their values at the surface of the underlying RSG models. In this work, we do not address the physical mechanism responsible for the formation of the CSM, which is crucial in defining the details of its structure. Instead, we concentrate on the general characteristics of the CSM, such as its total mass $M_{\mathrm{CSM}}$ and extent $R_{\text {ext }}$. Numerical studies of extended material around $\mathrm{SNe}$ demonstrate that the exact density distribution only of secondary importance to these main properties (e.g., Piro et al. 2017).

From the large set of progenitor models presented in Sukhbold et al. (2016), we choose a subset of models in the mass range between $9 M_{\odot}$ and $25 M_{\odot}$ in steps of $0.5 M_{\odot}$. Despite uncertainties in the upper limit (Smartt et al. 2009; Smith et al. 2011; Groh et al. 2013; Dwarkadas 2014), the stars in this range of ZAMS masses are believed to be pro-

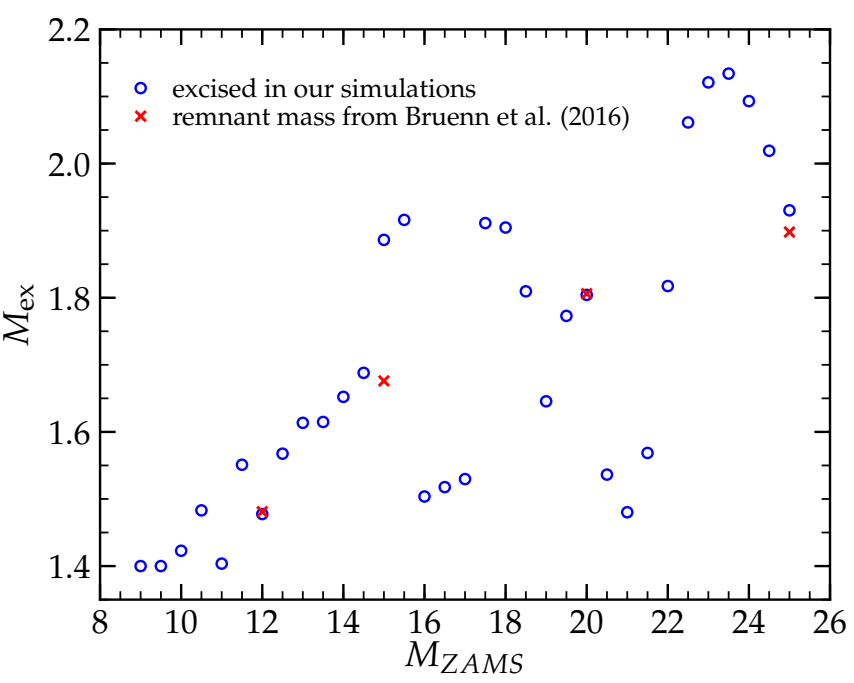

Figure 1. Excised mass as a function of ZAMS mass in our simulations (blue circles), which is taken to be equal to the mass coordinate of the siliconoxygen interface in the pre-collapse composition profile. For comparison, in red we show the baryonic rest masses of proto-neutron stars formed in corecollapse SN explosion mechanism simulations by Bruenn et al. (2016) using progenitor models of Woosley \& Heger (2007).

genitors of the bulk of Type II SNe. To obtain light curves from these models, we explode them with our open-source numerical code SNEC (Morozova et al. 2015) in the range of asymptotic explosion energies, $E_{\mathrm{fin}}$, between 0.04 and $1.3 \mathrm{~B}$ in steps of $0.02 \mathrm{~B}$, where $1 \mathrm{~B}=10^{51} \mathrm{erg}$. We use a thermal bomb mechanism for the explosions, where the thermal bomb energy, $E_{\mathrm{bomb}}$ is found from $E_{\mathrm{fin}}$ and the total pre-explosion (negative, mostly gravitational) energy of the model, $E_{\text {init }}$, as $E_{\text {bomb }}=E_{\text {fin }}-E_{\text {init }}$. This energy is then injected into the inner $0.02 M_{\odot}$ of the model for a duration of $1 \mathrm{~s}$. Previous work discusses the impact of this duration choice (Morozova et al. 2015, 2016, 2017). Exploding higher mass progenitors with low energies is challenging for our code, since the current version is not capable of treating material falling back onto a remnant. Therefore, there is a region of the parameter space not covered in our study (shown in Figure 4 as a gray shaded area).

Before exploding the models, we excise the inner part, which is assumed to form a neutron star. As shown in the large number of SN explosion mechanism simulations (e.g., Müller et al. 2012; Summa et al. 2016; Suwa et al. 2016; Burrows et al. 2016; Radice et al. 2017), the passage of the otherwise stalled shock wave through the density and composition discontinuities of the progenitor model, such as the boundary between the silicon- and oxygen- burning shells, can facilitate its revival and play an important role in the successful $\mathrm{SN}$ explosion. Therefore, one can expect the remnant masses to be equal or slightly larger than the mass coordinate of the $\mathrm{Si} / \mathrm{O}$ interface in the progenitor composition profile, and we use this as a criterium to determine the excised mass in our simulations. Figure 1 shows the excised mass as a function of ZAMS mass for our subset of models. The values of the baryonic rest mass of the proto-neutron stars from the simulations of Bruenn et al. (2016), using progenitor models from Woosley \& Heger (2007), are shown for comparison. Larger excision masses facilitate the explosion of large ZAMS mass progenitors to some extent. We note thought that as long as the explosion is successful, the light curve depends very weakly on the excised mass. 
In SNEC, we use the equation of state by Paczyński (1983) and solve for the ionization fractions of hydrogen and helium following the approach of Zaghloul et al. (2000). The numerical grid is identical to the one used in our previous studies and consists of 1000 cells (Morozova et al. 2015, 2016, 2017). We use the same prescription for the opacity floor as in those works, namely, $0.01 \mathrm{~cm}^{2} \mathrm{~g}^{-1}$ for the solar metallicity $Z=0.02,0.24 \mathrm{~cm}^{2} \mathrm{~g}^{-1}$ for $Z=1$, with the linear dependence in between. We smoothen the composition profiles before the explosion by passing a "boxcar" with a width of $0.4 M_{\odot}$ through the models four times. Photometric light curves are calculated assuming black body emission and using the MATLAB package for astronomy and astrophysics for calculating specific wave bands (Ofek 2014).

\section{NUMERICAL MODELS OF 20 SNE II}

\subsection{Observational data}

The SN sample we analyze here was chosen from a collection of high-quality SN II light curves presented in Valenti et al. (2016). The two main criteria we used in selecting these were (i) good multi-band light curve coverage and (ii) having an estimate of the ${ }^{56} \mathrm{Ni}$ mass from the radioactive tail. We have omitted a few SNe due to the large uncertainty in the explosion date ( $>6$ days) as well as the lack of data points during the transition between the plateau and the radioactive tail, both of which are important to obtain good quality fits using our approach.

Table 1 summarizes the observed properties of the selected SN sample. The distance modulus, DM, together with its uncertainty, $\triangle \mathrm{DM}$, are taken from Table 2 of Valenti et al. (2016). The interstellar and galactic values of the absorption in $B$-band, $A_{B, i}$ and $A_{B, g}$, respectively, are used to correct the light curves for reddening according to the Cardelli law (Cardelli et al. 1989) ${ }^{4}$. The plateau length $t_{\mathrm{PT}}$ is obtained by fitting a Fermi-Dirac function to the transition between the plateau and radioactive tail, and $\Delta t_{\mathrm{PT}}$ is its uncertainty ${ }^{5}$. The ${ }^{56} \mathrm{Ni}$ mass, $M_{\mathrm{Ni}}$, and its uncertainty, $\Delta M_{\mathrm{Ni}}$, were derived in Valenti et al. (2016) from the comparison of the post-plateau pseudo-bolometric light curves to the one of SN 1987A.

It has been highlighted in some observational work that some SN light curves demonstrate a change in slope at a few tens of days after the maximum (Anderson et al. 2014; Valenti et al. 2016). This change is more pronounced in the pseudobolometric light curves (including bands from $U / B$ to $I$ ) than in the single bands. The time $t_{\mathrm{S}}$ in Table 1 marks the last data point used to measure the early slope of the pseudobolometric light curves of the corresponding $\mathrm{SNe}$ in Valenti et al. (2016) (the first parameter ph_stop in their Table D4), which we use here as a proxy for the transition time between the early faster and the late shallow slopes.

\subsection{Two step approach to numerical modeling}

As discussed in Section 1, recent work demonstrates that a dense, compact CSM is crucial for modeling Type II SN light

\footnotetext{
${ }^{4}$ Note that the interstellar reddening shown in Table 1 was estimated based on the equivalent width of $\mathrm{Na}$ I D lines in the spectra of host galaxies and does not take into account possible extinction due to the circumstellar dust. However, since the circumstellar dust is likely destroyed by the SN explosion, it should not affect the light curve modeling. Negligible interstellar reddening for some $\mathrm{SNe}$ from the sample is additionally supported by the fact that they have similar colors during the plateau phase.

5 The values of $t_{\mathrm{PT}}$ are taken from Table D5 of Valenti et al. (2016) when available, otherwise from Table D4.
}

curves (Morozova et al. 2017). This means that to properly fit these light curves requires fitting for $M_{\mathrm{ZAMS}}, E_{\mathrm{fin}}, M_{\mathrm{Ni}}$, ${ }^{56} \mathrm{Ni}$ mixing, explosion time, $R_{\text {ext }}$, and $K$-a seven parameter fitting space! Since building such a larger grid of models is simply not feasible, we take advantage of the knowledge that the early light curve over the first $\sim 10-30$ days should be dominated by the CSM, and that the remainder of the light curve is dominated by the hydrogen-rich RSG envelope. This allows us to utilize a two step approach in fitting the SN light curves, which we describe further below.

In the first step of our light curve fitting, we generate a grid of light curves in $M_{\mathrm{ZAMS}}-E_{\mathrm{fin}}$ parameter space, in each case using the radioactive ${ }^{56} \mathrm{Ni}$ mass for each $\mathrm{SN}$ from Table 1. As was discussed in Section 2, we cover the parameter space $9 M_{\odot}<M_{\mathrm{ZAMS}}<25 M_{\odot}$ in steps of $0.5 M_{\odot}$ and $0.04 \mathrm{~B}<E_{\mathrm{fin}}<1.3 \mathrm{~B}$ in steps of $0.02 \mathrm{~B}$ (with the exception of the largest masses and smallest energies). To account for the effect of ${ }^{56} \mathrm{Ni}$ mixing into the envelope, we consider three degrees of ${ }^{56} \mathrm{Ni}$ mixing for each $\mathrm{SN}$, up to the mass coordinates of $3 M_{\odot}, 5 M_{\odot}$ and $7 M_{\odot}$ (in each case using a "boxcar" method as described above).

Within this grid, we look for the best fitting model for each SN by minimizing $\chi^{2}$, which we calculate as

$$
\chi^{2}=\sum_{\lambda \in[g, \ldots, z]} \sum_{t_{\mathrm{S}}<t^{*}<t_{\mathrm{PT}}} \frac{\left(M_{\lambda}^{*}\left(t^{*}\right)-M_{\lambda}\left(t^{*}\right)\right)^{2}}{\left(\Delta M_{\lambda}^{*}\left(t^{*}\right)\right)^{2}},
$$

where $M_{\lambda}^{*}\left(t^{*}\right)$ is the observed magnitude in a given band $\lambda$ at the moment of observation $t^{*}, \Delta M_{\lambda}^{*}\left(t^{*}\right)$ is the corresponding observational error, and $M_{\lambda}\left(t^{*}\right)$ is the numerically obtained magnitude in the same band at the same moment of time. To take into account uncertainties in the explosion date, we shift the time of observations within the allowed range of explosion times in steps of $0.5 \mathrm{~d}$, and look for the minimal $\chi^{2}$. In the first fitting step, we use only the parts of light curves between the time $t_{\mathrm{S}}$ and the end of plateau $t_{\mathrm{PT}}$. We do not go beyond $t_{\mathrm{PT}}$, since after this time the whole ejecta is expected to become optically thin and the diffusion approach to radiation transport used in SNEC is not valid anymore. We also do not include $u$-, $U$ - and $B$ - bands in Equation 2, since after day $\sim 20$ the radiation in these bands is affected by iron group line blanketing (Kasen \& Woosley 2009), which is not taken into account. As a result of this first step, we get the best fit values of progenitor ZAMS mass, explosion energy, degree of ${ }^{56} \mathrm{Ni}$ mixing and explosion time.

For the second step, we attach CSM on top of these best fitting RSG models, we generate a grid of light curves in $R_{\text {ext }}-K$ parameter space for each $\mathrm{SN}$. We vary $K$ in the range between $1.0 \times 10^{17}$ and $3.0 \times 10^{18}$ in steps of $1.0 \times 10^{17}$, and $R_{\text {ext }}$ in the range between $700 R_{\odot}$ and $3800 R_{\odot}$ in steps of $100 R_{\odot}$. We shift the observational data with respect to the explosion date in the same way that minimized $\chi^{2}$ during the first step. After that we assess the best fitting model within the $R_{\text {ext }}-K$ grid by calculating $\chi^{2}$ as we did in Equation 2, but for $t^{*}<t_{\mathrm{PT}}$ instead of $t_{\mathrm{S}}<t^{*}<t_{\mathrm{PT}}$. This second step results in the best fitting values of the CSM density parameter $K$ and external radius $R_{\text {ext }}$.

\subsection{Numerical results}

Figures 2 and 3 show all of the best fitting models for the $\mathrm{SNe}$ from our set, together with the observed light curves in different bands. The dashed lines show the result of the first fitting step (without CSM), while the solid lines show the final fit (with CSM). Residuals are shown to assess the quality 

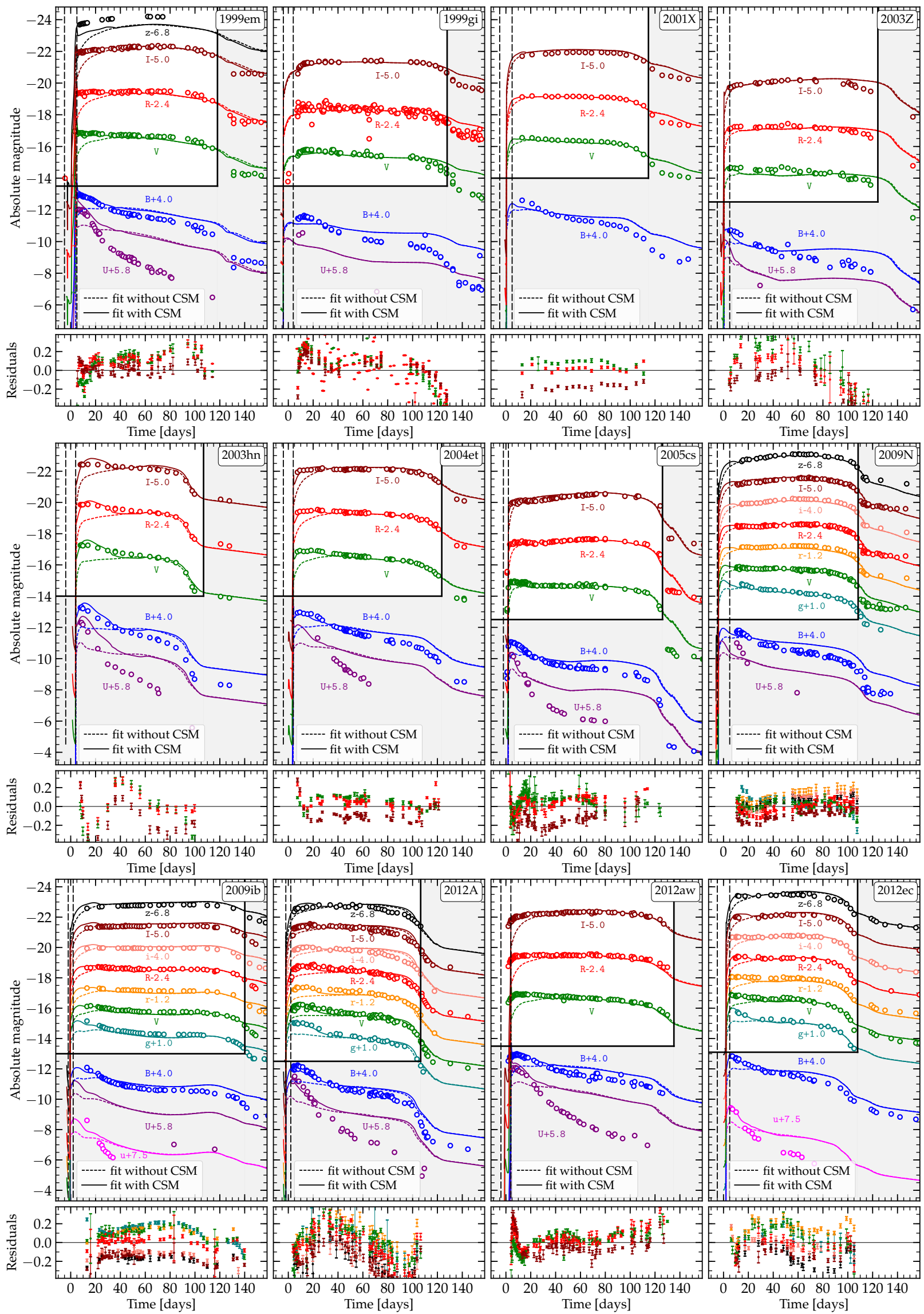

Figure 2. Best fit light curves with (solid lines) and without (dashed lines) CSM. Data is shown as open circles. Each color corresponds to a different wave band as labeled. Unshaded (white) regions contain the SN data used to find the fits while shaded (gray) regions are ignored for fitting either because it is too late in the light curve or iron group line blanketing may be important (in the bluer) bands. 
Table 1

Observed SN parameters.

\begin{tabular}{lccccccccc}
\hline \hline SN & DM & $\Delta \mathrm{DM}$ & $A_{B, i}[\mathrm{mag}]$ & $A_{B, g}[\mathrm{mag}]$ & $t_{\mathrm{PT}}[\mathrm{d}]$ & $\Delta t_{\mathrm{PT}}[\mathrm{d}]$ & $t_{\mathrm{S}}[\mathrm{d}]$ & $M_{\mathrm{Ni}}\left[M_{\odot}\right]$ & $\Delta M_{\mathrm{Ni}}\left[M_{\odot}\right]$ \\
\hline 1999em & 30.34 & 0.07 & 0.234 & 0.174 & 118.1 & 1.0 & 34.4 & 0.0536 & 0.0119 \\
1999gi & 30.34 & 0.14 & 0.0 & 0.07 & 127.8 & 3.1 & 38.2 & 0.0320 & 0.0023 \\
$2001 \mathrm{X}$ & 31.59 & 0.11 & 0.0 & 0.173 & 114.7 & 5.0 & 41.9 & 0.0550 & 0.0047 \\
$2003 Z$ & 31.70 & 0.15 & 0.0 & 0.141 & 124.2 & 4.5 & 29.4 & 0.0047 & 0.0002 \\
2003hn & 31.14 & 0.26 & 0.71 & 0.057 & 106.9 & 4.0 & 42.8 & 0.0324 & 0.0046 \\
2004et & 28.36 & 0.09 & 0.03 & 1.48 & 123.5 & 4.0 & 53.4 & 0.0414 & 0.0086 \\
2005cs & 29.26 & 0.33 & 0.0 & 0.205 & 126.0 & 0.5 & 22.3 & 0.0021 & 0.0002 \\
2009N & 31.67 & 0.11 & 0.532 & 0.078 & 108.3 & 1.2 & 26.6 & 0.0165 & 0.0021 \\
2009ib & 31.48 & 0.31 & 0.537 & 0.105 & 140.1 & 2.0 & 38.1 & 0.0520 & 0.0162 \\
2012A & 29.96 & 0.15 & 0.05 & 0.1 & 106.5 & 2.0 & 28.4 & 0.0087 & 0.0012 \\
2012aw & 29.96 & 0.09 & 0.24 & 0.115 & 135.2 & 4.0 & 36.6 & 0.0497 & 0.0059 \\
2012ec & 31.32 & 0.15 & 0.414 & 0.096 & 107.9 & 5.0 & 36.3 & 0.0394 & 0.0051 \\
2013ab & 31.90 & 0.08 & 0.081 & 0.099 & 101.8 & 1.0 & 36.4 & 0.0588 & 0.0100 \\
2013by & 30.81 & 0.15 & 0.0 & 0.798 & 85.4 & 2.0 & 39.7 & 0.0320 & 0.0043 \\
2013ej & 29.79 & 0.20 & 0.0 & 0.25 & 98.8 & 1.0 & 38.4 & 0.0207 & 0.0019 \\
LSQ13dpa & 35.08 & 0.15 & 0.0 & 0.137 & 128.7 & 2.0 & 36.9 & 0.0714 & 0.0127 \\
2014cy & 31.87 & 0.15 & 0.0 & 0.2 & 122.6 & 1.0 & 33.0 & 0.0037 & 0.0038 \\
ASASSN-14dq & 33.26 & 0.15 & 0.0 & 0.254 & 101.0 & 5.5 & 33.6 & 0.0461 & 0.0079 \\
ASASSN-14gm & 31.74 & 0.15 & 0.0 & 0.406 & 110.6 & 1.5 & 43.3 & 0.0767 & 0.0102 \\
ASASSN-14ha & 29.53 & 0.50 & 0.0 & 0.033 & 136.8 & 1.5 & 37.5 & 0.0014 & 0.0002 \\
\hline
\end{tabular}



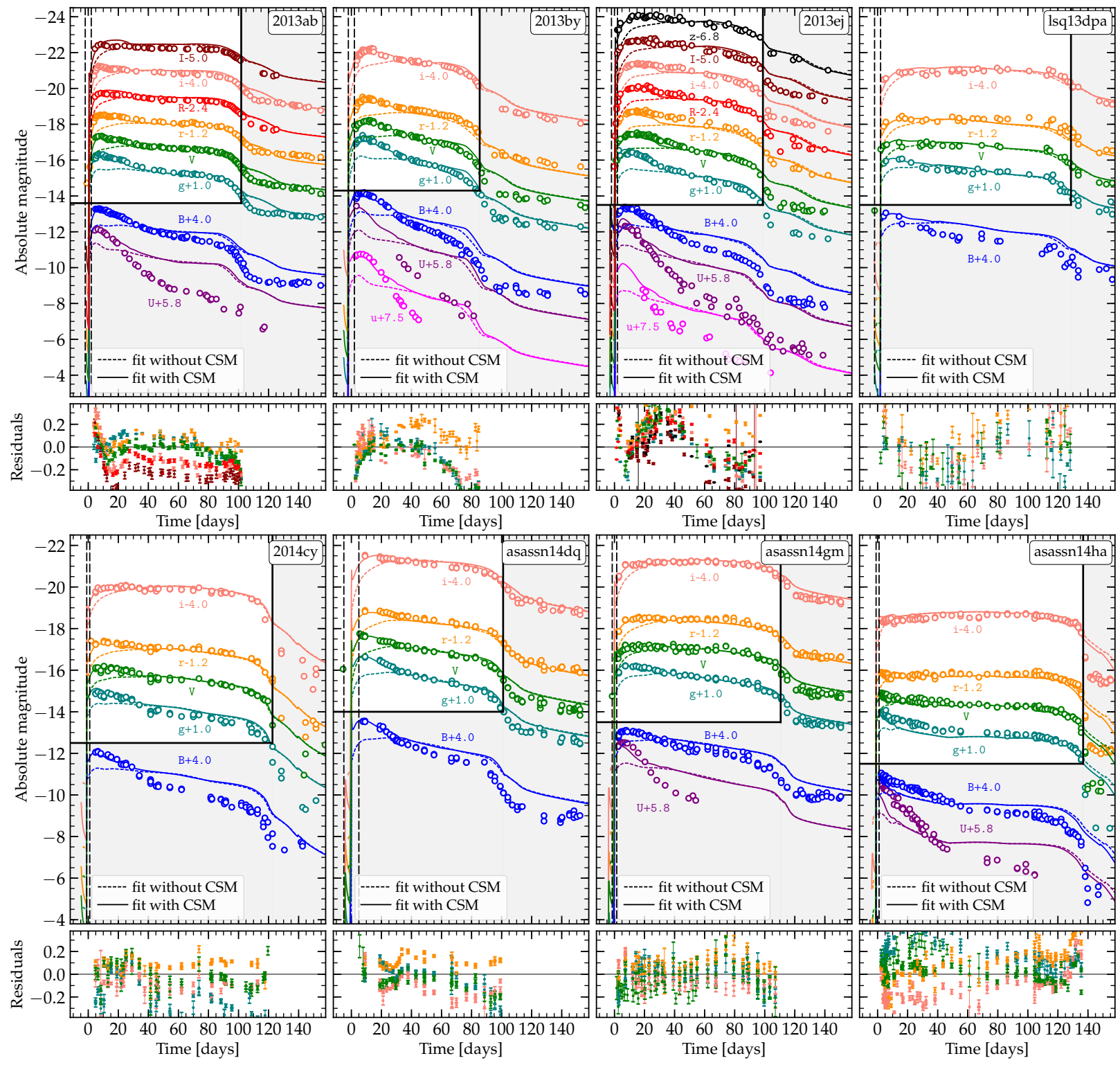

Figure 3. Additional $\mathrm{SNe}$, the same as Figure 2.

of the fits. The shaded regions in the plots contain the data that were not used in our analysis for the reasons described in Section 3.2. For completeness, we partially show the radioactive tails of our light curves. We emphasize, however, that our code uses diffusion approach for the radiation transport, which works well for the shock cooling and plateau part of the light curve, but is not suitable for the nebular phase. In addition, as described in the code manual ${ }^{6}$, for the purpose of magnitude calculations the effective temperature is kept above $5000 \mathrm{~K}$, following the reasoning of Swartz et al. (1991). Therefore, no conclusion concerning the goodness of the fit can be made based on data after the end of plateau.

As emphasized in previous work (Morozova et al. 2017), we find that light curves obtained from the bare RSGs are significantly poorer at fitting the data in comparison to light curves including dense CSM. The dashed curves in Figures 2 and 3 rise more slowly than the observations during the first

6 https://stellarcollapse.org/codes/snec_notes-1. $00 \cdot p d f$
20 - 40 days (similar to Figures 2-4 of Morozova et al. 2017). On the other hand, if one were to restrict the fitting window for these models to just the time between the slope break and the end of plateau, then reasonable fits can be made with just bare RSGs. This lends support for the two step method we utilized for the light curve fitting. Interestingly, the addition of dense CSM even improves the fit of the early light curve in the $B$ and $U$ bands that we do not include when fitting. This is not surprising given that at the high effective temperatures of the early light curves $(10,000 \mathrm{~K}$ and higher) the iron group line blanketing does not play an important role and the spectrum is very close to a black body. Nevertheless, this is further evidence for the necessary presence of dense CSM.

Figures 4 and 5 illustrate the dependence of $\chi^{2}$ on the grid parameters. Since the minimum of $\chi^{2}$ in our models is rather shallow, it does not make much sense to access the confidence regions as we previously did in Morozova et al. (2017), because the $39.3 \%$ confidence region will cover $\sim 39.3 \%$ of the domain, etc. Instead, we color code the natural logarithm of $\chi^{2} / \chi_{\min }^{2}$, so that the yellow contour in the figures approxi- 


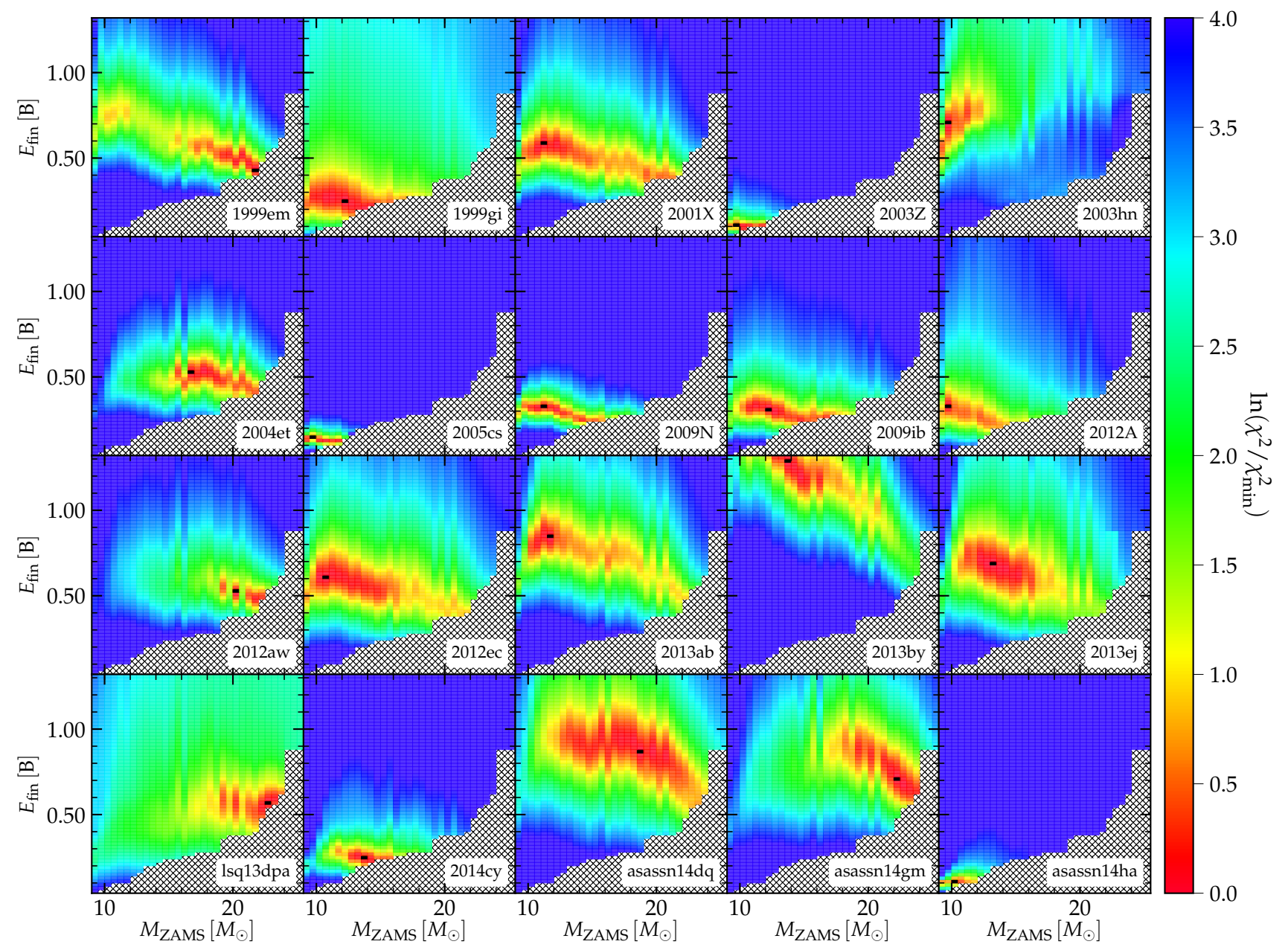

Figure 4. Color coded ratio of $\chi^{2}$ to $\chi_{\min }^{2}$ at each grid point in $M_{\mathrm{ZAMS}}-E_{\mathrm{fin}}$ space. Yellow contour approximately corresponds to the $\chi^{2}$ increase by a factor of $e$ with respect to the minimum. The black square in each panel indicates the best fitting parameters. Gray shaded regions could not be covered by the current study due to the numerical difficulties. For each SN, the plotted $\chi^{2}$ corresponds to the best fitting degree of ${ }^{56} \mathrm{Ni}$.

mately corresponds to the increase of $\chi^{2}$ by a factor of $e$ with respect to the minimum, while the green region corresponds to an order of magnitude increase. We adopt the extents of the yellow contours as the error estimates of our fits. Shaded gray regions in Figure 4 indicate the part of the domain which could not be covered by the light curves due to numerical difficulties, while in Figure 5 they just cut away the regions smaller than the radii of the underlying RSGs.

From Figure 4 one can see that the explosion energy is generally better constrained by our models than the progenitor ZAMS mass. The pre-explosion profiles of the progenitors are determined by complex nuclear burning processes during stellar evolution and do not always demonstrate smooth dependence on the ZAMS mass, which results in striped patterns in Figure 4. Figure 5 demonstrates a strong degeneracy between the density and the radial extent of the CSM. It is interesting to note, however, that the contours of constant $\chi^{2}$ follow very closely the contours of constant CSM mass, which we plotted in the figure with white dashed lines. This tells us that the total mass of the CSM can be inferred from our fits more robustly than its density and external radius separately (as has been found for extended material in other types of SNe, Piro et al. 2017). As for the CSM radius, it is only a few times the RSG radius in all cases. This indicates that whatever causes the mass loss must happen at the very last stages of the RSG's life.

All fitting parameters are summarized in Table 2. The different degrees of ${ }^{56} \mathrm{Ni}$ mixing result in rather close, and in some cases even identical, fitting progenitor characteristics (the best fitting model among the three mixing degrees is shown in bold). The energy released in the radioactive decay of ${ }^{56} \mathrm{Ni}$ is expected to prolong the plateau and/or flatten it (Nakar et al. 2016). In our grid, though, this subtle effect gets smeared by others, like discontinuities in the properties of the progenitor models themselves, and the difference in fitting parameters between the different mixing degrees is smaller than the error bars we estimate based on Figure 4.

As for the CSM, we added to the table its total mass $M_{\mathrm{CSM}}$, without trying to convert it into the mass losses in $M_{\odot} \mathrm{yr}^{-1}$, which an interested reader can easily estimate from Equation (1) for a reasonable wind speed. In fact, there is no reason to assume a wind speed of $10 \mathrm{~km} \mathrm{~s}^{-1}$, which is commonly observed in steady state RSG winds. Higher wind velocities of $\sim 100 \mathrm{~km} \mathrm{~s}^{-1}$ in our models would correspond to larger mass loss rates of the order of few $M_{\odot} \mathrm{yr}^{-1}$ and the duration of the enhanced mass loss period of only few months before explosion. However, since our models are not sensitive to the wind velocity in the pre-explosion models, but rather to the density profile of the wind, we cannot put any constraint on the mass loss rate and the duration of the outflow. Moreover, we em- 


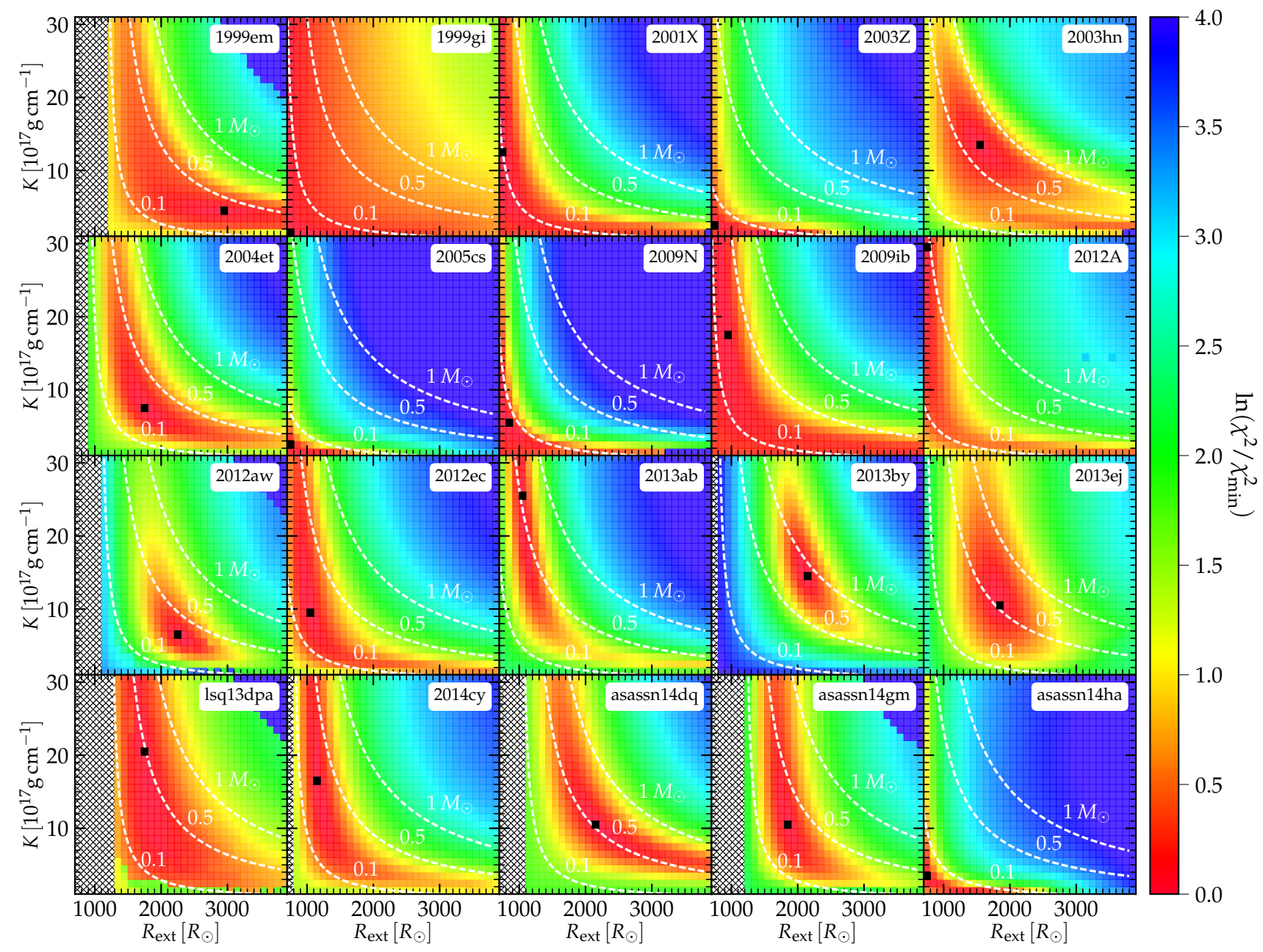

Figure 5. Color coded ratio of $\chi^{2}$ to $\chi_{\min }^{2}$ at each grid point in $R_{\text {ext }}-K$ space. Yellow contour approximately corresponds to the $\chi^{2}$ increase by a factor of $e$ with respect to the minimum. The black square in each panel indicates the best fitting parameters. Shaded with gray are the regions below the RSG radius of the best fitting model for the corresponding SNe. White lines show the constant CSM mass contours $\left(0.1 M_{\odot}, 0.5 M_{\odot}\right.$ and $\left.1 M_{\odot}\right)$.

phasize that it is entirely possible that the dense CSM is not exactly a wind but a different density distribution for which the wind profile we use is an approximation. The origin of the CSM is an interesting topic of investigation on its own, and it lays beyond the scope of this study, although we discuss it further below.

It is worth noting that in Morozova et al. (2017) we also modeled two SNe from the current sample, 2013ej and 2013 by, but using a slightly different approach. There we generated entire 4-dimensional grids of light curves in $M_{\mathrm{ZAMS}}$, $E_{\text {fin }}, K, R_{\text {ext }}$ parameter space, instead of using the twostep fitting procedure described here. This previous approach was more computationally expensive, which translated to a coarser resolution of these parameters. In Morozova et al. (2017), for SN 2013ej we got the values of $M_{\mathrm{ZAMS}}=$ $12.5 M_{\odot}, E_{\text {fin }}=0.6 \mathrm{~B}, K=1.0 \times 10^{18} \mathrm{~g} \mathrm{~cm}^{-1}, R_{\text {ext }}=$ $2100 R_{\odot}$, and for SN 2013by we got $M_{\mathrm{ZAMS}}=14.5 M_{\odot}$, $E_{\text {fin }}=1.4 \mathrm{~B}, K=1.0 \times 10^{18} \mathrm{~g} \mathrm{~cm}^{-1}, R_{\text {ext }}=2300 R_{\odot}$, both of which are in good agreement with the values from Table 2 . This adds additional credence to the scheme we use in the current work. Unfortunately, we could not fit SN 2013fs using the two-step approach because of the lack of data near the transition between the plateau and radioactive tail.

\section{DISCUSSION}

With the results from Section 3.3, we have for the first time ZAMS masses and dense CSM properties for a collection of Type II SNe based solely on light curve fitting. This allows us to compare the parameters we measure with other studies in the literature using different methods to constrain the properties of SN II progenitors. Furthermore, we can look for correlations between the various parameters with the hope of getting a better understanding of the explosion mechanism as well as the origin of the dense CSM. We discuss each of these comparisons below.

\subsection{Ejecta masses}

Figure 6 compares the ejecta masses and explosion energies obtained in our work to the other recently published studies of Utrobin \& Chugai (2013) and Pumo et al. (2017) (who, together with their own, collect the results of Tomasella et al. 2013; Spiro et al. 2014; Dall'Ora et al. 2014; Takáts et al. 2014, 2015; Bose et al. 2015; Huang et al. 2015). This figure contains only the values obtained from the hydrodynamical models of the corresponding events, and does not include the numerous estimates obtained from the analytical scalings, spectra or X-ray/radio signals (see, among many others, Misra et al. 2007; Jerkstrand et al. 2012, 2014; Chakraborti et al. 2016; Yuan et al. 2016; Dhungana et al. 2016). These values are collected in the last column of Table 2. Some of the SNe 
Table 2

Best fit parameters. Bold parameters highlight those with the smallest $\chi^{2}$ among the three degrees of ${ }^{56} \mathrm{Ni}$ mixing. These values are shown in Figures $2-5$ of Section 3 and used for the analysis of Section 4. All masses and radii are given in solar units, energies in B and parameter $K$ in $\mathrm{g} \mathrm{cm}^{-1}$. The last column lists the values used in Figure 6 only and not meant to fully represent the numerous literature (see more references in the text).

\begin{tabular}{|c|c|c|c|c|c|c|c|c|c|c|c|}
\hline \multirow[b]{2}{*}{ SN } & \multicolumn{2}{|c|}{${ }^{56} \mathrm{Ni}$ mixed to $3 M_{\odot}$} & \multicolumn{2}{|c|}{$\overline{56} \mathrm{Ni}$ mixed to $5 M_{\odot}$} & \multicolumn{2}{|c|}{$\overline{56} \mathrm{Ni}$ mixed to $7 M_{\odot}$} & \multicolumn{3}{|c|}{ CSM parameters } & \multicolumn{2}{|c|}{ Best fit $M_{\mathrm{ej}}$} \\
\hline & $M_{\mathrm{ZAMS}}$ & $E_{\text {fin }}$ & $M_{\mathrm{ZAMS}}$ & $E_{\text {fin }}$ & $M_{\mathrm{ZAMS}}$ & $E_{\text {fin }}$ & $K$ & $R_{\text {ext }}$ & $M_{\mathrm{CSM}}$ & This work & Other works \\
\hline 1999em & 21.5 & 0.42 & 20.5 & 0.52 & 20.0 & 0.48 & $4.0 \times 10^{17}$ & 2900 & 0.31 & 14.48 & $19^{a}$ \\
\hline 1999gi & 12 & 0.24 & 12 & 0.22 & 10.5 & 0.22 & $1.0 \times 10^{17}$ & 700 & $<0.003$ & 9.42 & - \\
\hline $2001 X$ & 11 & 0.58 & 12 & 0.5 & 12.0 & 0.46 & $1.2 \times 10^{18}$ & 700 & $<0.07$ & 9.29 & - \\
\hline $2003 Z$ & 9.5 & 0.1 & 10 & 0.08 & 10 & 0.08 & $2.0 \times 10^{17}$ & 700 & $<0.03$ & 7.81 & $14^{a}, 11.3^{b}$ \\
\hline $2003 \mathrm{hn}$ & 9.5 & 0.76 & 9.5 & 0.7 & 9.5 & 0.66 & $1.3 \times 10^{18}$ & 1500 & 0.63 & 7.81 & - \\
\hline 2004et & 20 & 0.42 & 18 & 0.54 & 16.5 & 0.52 & $7.0 \times 10^{17}$ & 1700 & 0.25 & 12.47 & $22.9^{a}$ \\
\hline $2005 \mathrm{cs}$ & 9.5 & 0.14 & 9.5 & 0.14 & 9.5 & 0.14 & $2.0 \times 10^{17}$ & 700 & $<0.03$ & 7.81 & $15.9^{a}, 9.5^{c}$ \\
\hline $2009 N$ & 10 & 0.34 & 11 & 0.34 & 11 & 0.32 & $5.0 \times 10^{17}$ & 800 & 0.05 & 9.29 & $11.5^{d}$ \\
\hline $2009 \mathrm{ib}$ & 12 & 0.3 & 14.5 & 0.24 & 12.5 & 0.24 & $1.7 \times 10^{18}$ & 900 & 0.2 & 9.42 & $15^{e}$ \\
\hline $2012 \mathrm{~A}$ & 9.5 & 0.32 & 9.5 & 0.3 & 9.5 & 0.3 & $2.9 \times 10^{18}$ & 700 & $<0.38$ & 7.81 & $12.5^{f}$ \\
\hline 2012aw & 20 & 0.52 & 20 & 0.52 & 20 & 0.52 & $6.0 \times 10^{17}$ & 2200 & 0.3 & 14.04 & $19.6^{g}$ \\
\hline $2012 \mathrm{ec}$ & 10.5 & 0.68 & 10.5 & 0.64 & 10.5 & 0.6 & $9.0 \times 10^{17}$ & 1000 & 0.18 & 8.71 & - \\
\hline 2013ab & 11.5 & 0.84 & 12 & 0.76 & 11.5 & 0.7 & $2.5 \times 10^{18}$ & 1000 & 0.48 & 9.20 & $7^{h}$ \\
\hline 2013by & 13.5 & 1.28 & 12.5 & 1.3 & 12.5 & 1.28 & $1.4 \times 10^{18}$ & 2100 & 0.83 & 10.16 & - \\
\hline $2013 \mathrm{ej}$ & 13 & 0.68 & 13.5 & 0.66 & 13 & 0.66 & $1.0 \times 10^{18}$ & 1800 & 0.49 & 9.95 & $10.6^{i}$ \\
\hline LSQ13dpa & 20 & 0.56 & 20 & 0.56 & 22.5 & 0.56 & $2.0 \times 10^{18}$ & 1700 & 0.43 & 14.30 & - \\
\hline 2014 cy & 13.5 & 0.24 & 13.5 & 0.24 & 13.5 & 0.24 & $1.6 \times 10^{18}$ & 1100 & 0.25 & 10.16 & - \\
\hline ASASSN-14dq & 19.5 & 0.86 & 18.5 & 0.86 & 18.5 & 0.86 & $1.0 \times 10^{18}$ & 2100 & 0.48 & 13.13 & - \\
\hline ASASSN-14gm & 23.5 & 0.62 & 23.5 & 0.62 & 22 & 0.7 & $1.0 \times 10^{18}$ & 1800 & 0.27 & 14.40 & - \\
\hline ASASSN-14ha & 10 & 0.1 & 10 & 0.1 & 10 & 0.1 & $3.0 \times 10^{17}$ & 700 & $<0.03$ & 8.25 & - \\
\hline
\end{tabular}

that are common between samples are marked with special symbols.

For almost all of the SNe (with the exception of SN 2013ab) we get lower ejecta masses and energies than the previous work. At least part of this difference comes from us using stellar evolution progenitor models instead of the double polytropic models widely used in the literature. The difference between evolutionary and non-evolutionary models that are capable of fitting the observational data equally well has been investigated by Utrobin et al. (2017) for SN 1999em. We add to this by comparing non-evolutionary and evolutionary models for SNe 2005cs and 2004et in Figure 7. For the evolutionary profiles we show our best fit models from Section 3, while the polytropic models are from Utrobin \& Chugai (2008) and Utrobin \& Chugai (2009). It is seen from Figure 7 that the non-evolutionary models predict considerably higher progenitor masses than the evolutionary models. It is known from the analytical scalings (Arnett 1980; Chugai 1991; Popov 1993), that there is a certain degeneracy in the way the ejecta mass and the explosion energy influence the luminosity and duration of the light curve. Increasing the explosion energy makes the plateau shorter but more luminous, and so does decreasing the ejecta mass. This explains why more massive nonevolutionary progenitors also require higher explosion energies in order to reproduce the observed light curves. It is worth noticing that the difference in the fitting models shown in Figure 7 is not likely due to the difference in the numerical codes, because with SNEC we could successfully reproduce the fit of SN 1999em from Bersten et al. (2011) (very similar to the one from Utrobin 2007), using the same double polytropic model (see Appendix A of Morozova et al. 2015).

Our results from Figure 6 show less of a correlation between ejecta mass and explosion energy, but at the same time,

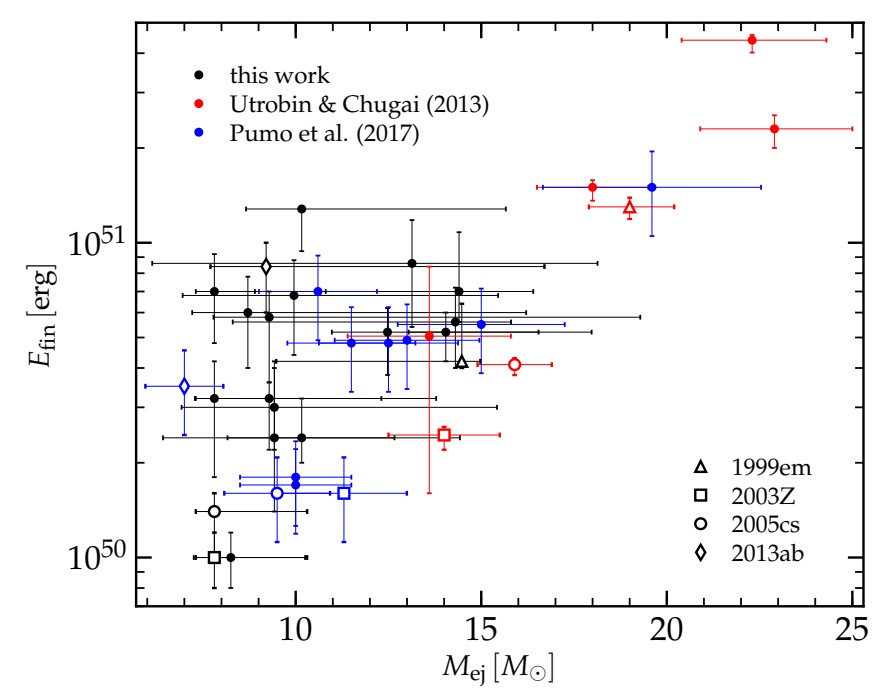

Figure 6. Ejecta masses versus explosion energies derived for our sample of $20 \mathrm{SNe}$ (black symbols), compared to the results of previous works. The error bars for our values are estimated based on the extent of the yellow contours in Figure 4, for Utrobin \& Chugai (2013) based on their Figure 8, and for Pumo et al. (2017) taken to be $30 \%$ of the value of energy and $15 \%$ of the value of mass, as suggested by the authors.

all three samples show a lack of high ejecta masses with low energies. Although it is true that we did not consider some regions of this parameter space due to the numerical issues, from Figure 4 it is seen that most of the best fitting models are not near this boundary of the modeled region. Earlier, correlation between the progenitor mass and the explosion energy was studied, for example, in Poznanski (2013).

\subsection{Progenitor ZAMS masses}




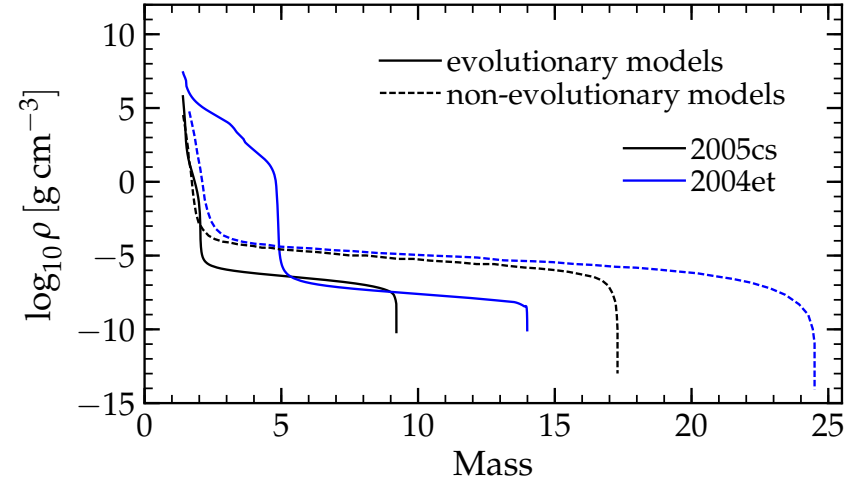

Figure 7. Comparison of the evolutionary and non-evolutionary models that produce numerical light curves (using different explosion energies) that compare favorably to the observations of SNe 2005cs and 2004et.

It is interesting to examine our results in view of the RSG problem, first described in Smartt et al. (2009). The problem is that analysis of the archival pre-explosion images of SNe IIP implies an upper limit of $\sim 17 M_{\odot}$ for the ZAMS mass of their progenitors. This is significantly lower than the expected limit of $\sim 25 M_{\odot}$, obtained from the comparison of the observed properties of RSGs with their possible evolutionary tracks (Levesque et al. 2005, 2006). In addition, models of light curves of the same $\mathrm{SNe}$ are known to predict higher progenitor masses than those derived from the direct imaging (Utrobin \& Chugai 2008, 2009).

The reason for this discrepancy may lie either on the observational side, on the theoretical side, or both. On the observational side, Walmswell \& Eldridge (2012) proposed that extinction due to the circumstellar dust helps to increase the progenitor masses derived from the pre-explosion images. However, in the subsequent work, Kochanek et al. (2012) demonstrated that the effects of dust composition, photon scattering and near-IR dust emission may instead lead to an even lower progenitor mass estimate. On the theoretical side, the problem may come from the progenitor models or various physical approximations used in the codes. Finally, it is possible that stars with ZAMS masses in the range $17-25 M_{\odot}$ may evolve past RSG stage due to enhanced mass loss or binary interactions and eventually explode as $\mathrm{SNe}$ of other types rather normal Type II (IIb/n or even Ib/c; see Smith et al. 2011).

The upper limit for the ZAMS mass of SNe II progenitors could signify that more massive stars collapse to black holes. They might eject their hydrogen envelope in a low energy explosion (as described in the work of Nadezhin 1980; Piro 2013; Lovegrove \& Woosley 2013; Lovegrove et al. 2017 and potentially observed by Adams et al. 2017) rather than a typical SN. Such a process would then imply that the helium core masses of these stars become the black hole masses (Kochanek 2014; Clausen et al. 2015). Therefore the inferred maximum mass for SN II progenitors may or may not explain the observed distribution of Galactic black hole masses (Özel et al. 2010) and the origin of a potential mass gap between neutron stars and black holes (Özel et al. 2012).

The top panel of Figure 8 shows $8 \mathrm{SNe}$ from our sample that also have an estimate of the progenitor ZAMS mass obtained from the pre-explosion imaging. All values shown along the $x$-axis of Figure 8 are taken from the recent work of Davies \& Beasor (2018), which summarizes and corrects the previous pre-explosion imaging mass estimates using updated bolometric corrections for RSGs. Earlier analysis of the pre-explosion images for the SNe shown in Figure 8 may be found in Walmswell \& Eldridge (2012); Tomasella et al. (2013); Maund et al. (2013, 2014); Fraser et al. (2014); Fraser (2016); Kochanek et al. (2012) and Mauerhan et al. (2017). We use filled symbols for SNe with direct progenitor detections and the empty symbols for SNe that only have upper limits due to a non-detection of the progenitor.

At a first glance, the agreement between the masses obtained from the pre-explosion imaging and the hydrodynamical modeling in Figure 8 does not look good. However, it is important to note that these two parts of the analysis currently use progenitor models from different stellar evolution codes (STARS is used in Davies \& Beasor (2018), and KEPLER in our work). The progenitor models from these two evolutionary codes are compared in Figure 5 of Jerkstrand et al. (2014), which demonstrates that for the same pre-explosion luminosity the STARS models predict systematically lower ZAMS masses. The difference between the STARS and KEPLER masses is about $1-2 M_{\odot}$ in the lower ZAMS mass range $\left(10-15 M_{\odot}\right)$ and up to $4 M_{\odot}$ in the higher ZAMS mass range $\left(20-25 M_{\odot}\right)$. Were the ZAMS masses in Figure 8 shifted correspondingly to the right along the $x$-axis, this would improve the agreement with the results of our modeling. We note that our $M_{\mathrm{ZAMS}}$ values for most of the $\mathrm{SNe}$ are lower than some of the other ones previously obtained in the literature (Maguire et al. 2010; Dall'Ora et al. 2014).

The bottom panel of Figure 8 shows 5 SNe from our sample that have an estimate of the progenitor ZAMS mass obtained from the analysis of the surrounding stellar populations by Maund (2017). In this panel, SNe 2013ej and 2004et show better agreement with the numerical models than in the top panel. An interesting exception is SN 2012ec, for which the analysis of both pre-explosion images and stellar populations predicts considerably higher ZAMS mass than the numerical modeling.

We next explore what ZAMS mass distribution is implied by our sample. To do this we consider a Salpeter initial mass function (IMF) and investigate what lower and upper limits on the progenitor ZAMS mass, $M_{\min }$ and $M_{\max }$, respectively, are needed to match our distribution. Following Smartt et al. (2009), we calculate the probability function for each SN in Table 2 as

$$
\begin{aligned}
P_{j}= & \int_{M_{j, \text { low }}}^{M_{j}} \frac{\left(M-M_{j, \text { low }}\right) M_{j}^{\Gamma-1}}{\left(M_{j}-M_{j, \text { low }}\right)\left(M_{\min }^{\Gamma}-M_{\max }^{\Gamma}\right)} d M \\
& +\int_{M_{j}}^{M_{j, \text { high }}} \frac{M^{\Gamma-1}}{\left(M_{\text {min }}^{\Gamma}-M_{\text {max }}^{\Gamma}\right)} d M,
\end{aligned}
$$

where $M_{j}$ is the best fitting ZAMS mass of the $j$-th SN, $M_{j \text {,low }}$ and $M_{j \text {,high }}$ are its lower and upper uncertainties, and $\Gamma=-1.35$ for the Salpeter initial mass function (IMF). When $M_{j \text {,low }}$ or $M_{j \text {,high }}$ are higher or lower than $M_{\min }$ or $M_{\max }$, we use the latter as the integration limits. The maximum of the likelihood, calculated as $\mathcal{L}=\prod P_{j}$, corresponds to $M_{\min }=10.4 M_{\odot}$ and $M_{\max }=22.9 M_{\odot}$, as shown in black in the top panel of Figure 9. The 68, 90 and $95 \%$ confidence regions are estimated from the condition

$$
\ln \mathcal{L}_{\max }-\ln \mathcal{L}=\frac{1}{2} \chi,
$$

where $\chi=2.3,4.6$ and 6.2 , correspondingly. The bottom panel of Figure 9 shows the cumulative frequency plot of the ZAMS masses derived from the numerical fit, analogous to the plot first published in Smartt et al. (2009) for the ZAMS 

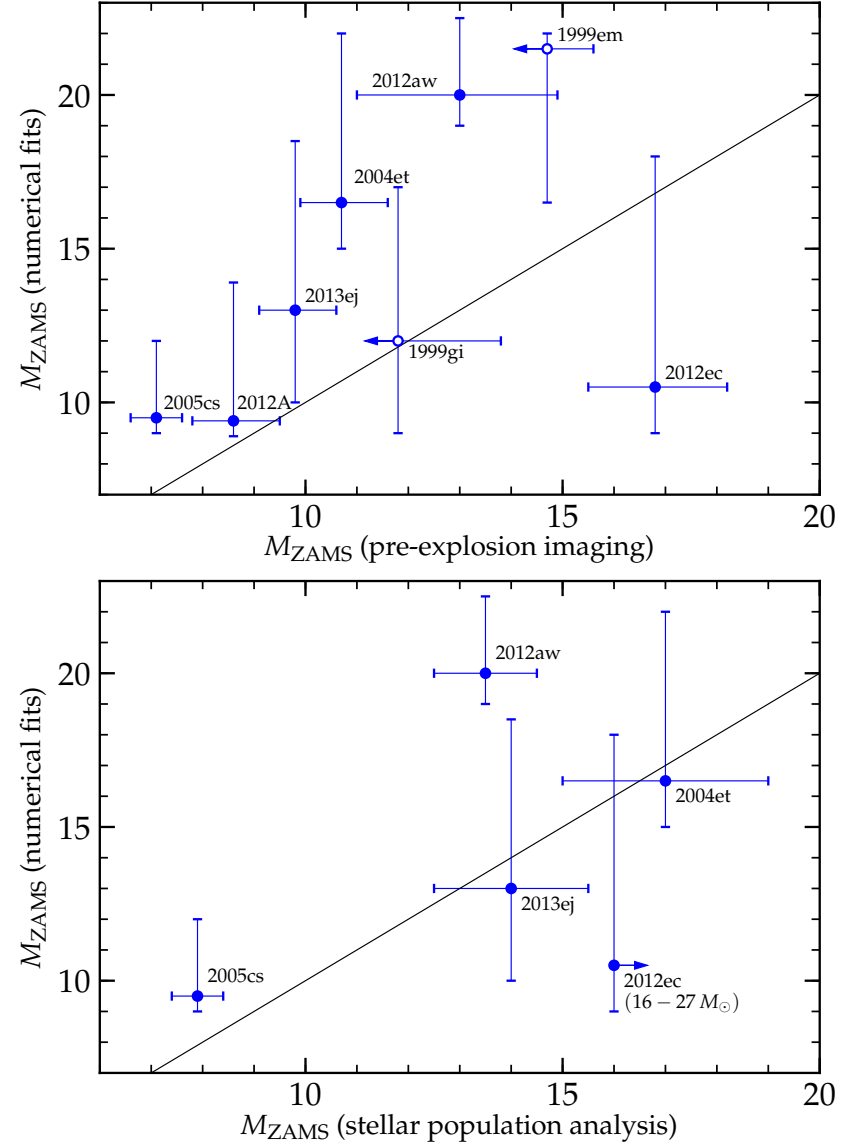

Figure 8. Top panel: Comparison of ZAMS masses of $8 \mathrm{SNe}$ obtained from our numerical fits with those obtained with the pre-explosion imaging (Davies \& Beasor 2018, filled symbols indicate the progenitor detections, while empty symbols with arrows indicate upper limits from progenitor nondetections). Poor agreement between the ZAMS masses may be at least partly explained by the difference between the progenitor models from STARS and KEP LER evolutionary codes (Jerkstrand et al. 2014, see the discussion in the text). Bottom panel: Comparison of ZAMS masses of SNe obtained from our numerical fits with those obtained from the analysis of the surrounding stellar populations by Maund (2017).

masses derived from the pre-explosion images (their Figure 8 ). The solid line shows the Salpeter IMF with the minimum mass of $10.4 M_{\odot}$ and the maximum mass of $22.9 M_{\odot}$, derived from maximizing the likelihood.

For comparison with the pre-explosion imaging, in the top panel of Figure 9 we show the values for maximum and minimum masses obtained in Davies \& Beasor (2018), using STARS and KEPLER stellar evolution codes. This plot demonstrates that using the same set of progenitor models for the pre-explosion imaging and the hydrodynamical modeling improves the agreement between the mass limits. The maximum mass that we obtain is closer to the maximum mass of RSGs $\left(25-30 M_{\odot}\right)$ seen from observations (Massey et al. 2001; Levesque et al. 2005, 2006; Crowther 2007). It therefore appears, coming back to the discussion about RSGs above, that our distribution of masses relieves some of the tension in the RSG problem.

\subsection{SN explosion properties}

It is natural to assume that there must be a correlation between the explosion energy and the ${ }^{56} \mathrm{Ni}$ mass since this is synthesized as the shock propagates out through the dense regions of the core (Nadyozhin 2003). This correlation has al-
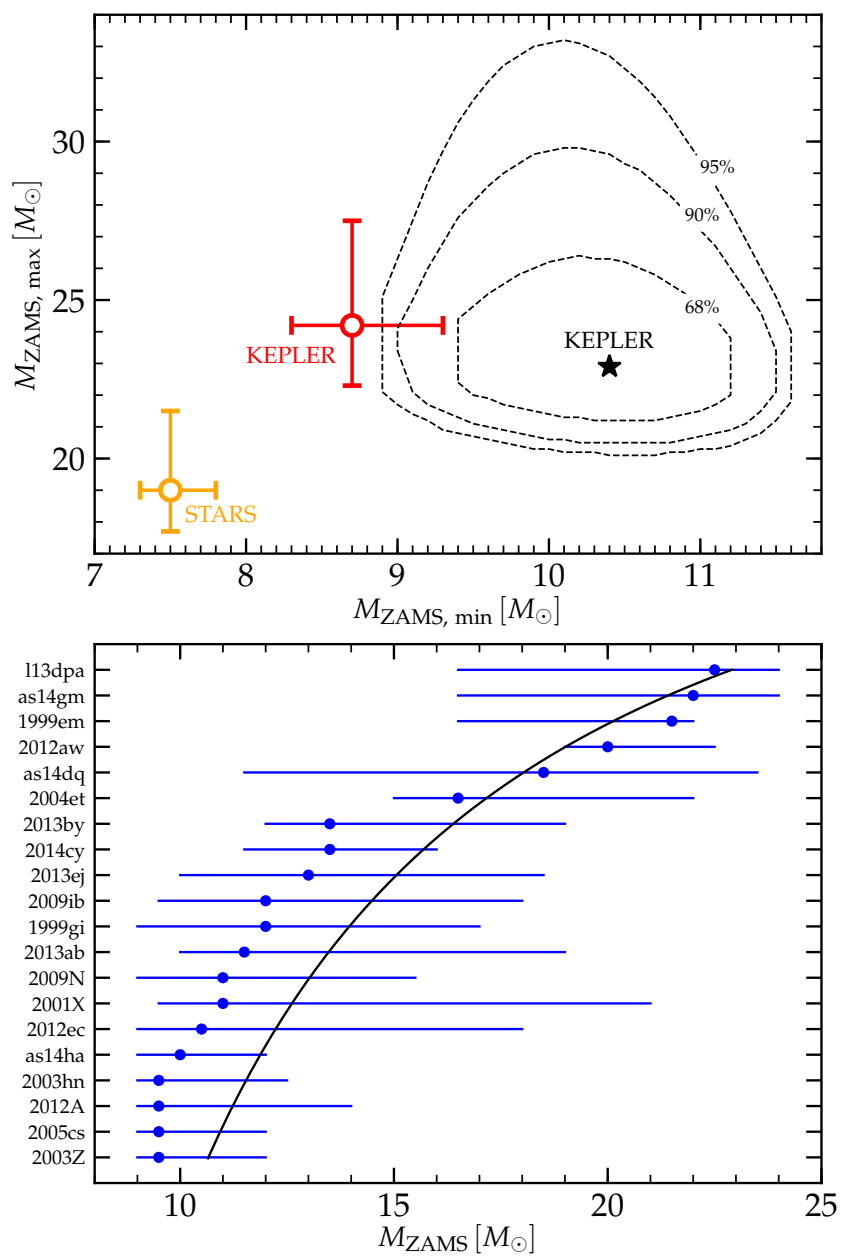

Figure 9. Top panel: The likelihood function for the minimum and maximum ZAMS masses of the Type II SN progenitors. The maximum likelihood derived from our numerical fits corresponds to $M_{\mathrm{ZAMS} \text {, min }}=10.4 M_{\odot}$ and $M_{\mathrm{ZAMS}, \max }=22.9 M_{\odot}$, indicated by a black star in the plot. The contours show the 68,90 and $95 \%$ confidence regions. For comparison, in yellow and red we show the values obtained in Davies \& Beasor (2018) from the pre-explosion imaging, using STARS and KEP LER stellar evolution codes, respectively. The estimates obtained with the same evolutionary code demonstrate better agreement, supporting our earlier discussion on Figure 8. Bottom panel: The cumulative frequency plot of the ZAMS masses derived from the numerical fits. The black line shows a Salpeter IMF with minimum and maximum ZAMS masses of 10.4 and $22.9 M_{\odot}$, respectively.

ready been seen by the nucleosynthetic simulations (Sukhbold et al. 2016) and light curve parameterization methods (Pejcha \& Thompson 2015). Furthermore, observations show this correlations through the ${ }^{56} \mathrm{Ni}$ mass and the plateau magnitudes of SNe II (Hamuy 2003; Spiro et al. 2014; Valenti et al. 2016) as well as their ejecta velocities (Maguire et al. 2012). Here we test whether this correlation is also seen simply from our light curve fitting. Figure 10 compares the explosion energy derived from the numerical fits and the ${ }^{56} \mathrm{Ni}$ mass of the corresponding $\mathrm{SNe}$. This shows a strong correlation between the two, similar to these other previous studies.

\subsection{CSM properties}

With our sample of SNe and models we can next address whether the CSM mass and radii we infer correlate with other properties of the SN or progenitor. This can hopefully provide clues about the mechanism by which the dense CSM we are finding is generated.

Figure 11 shows the derived CSM masses versus the ZAMS 


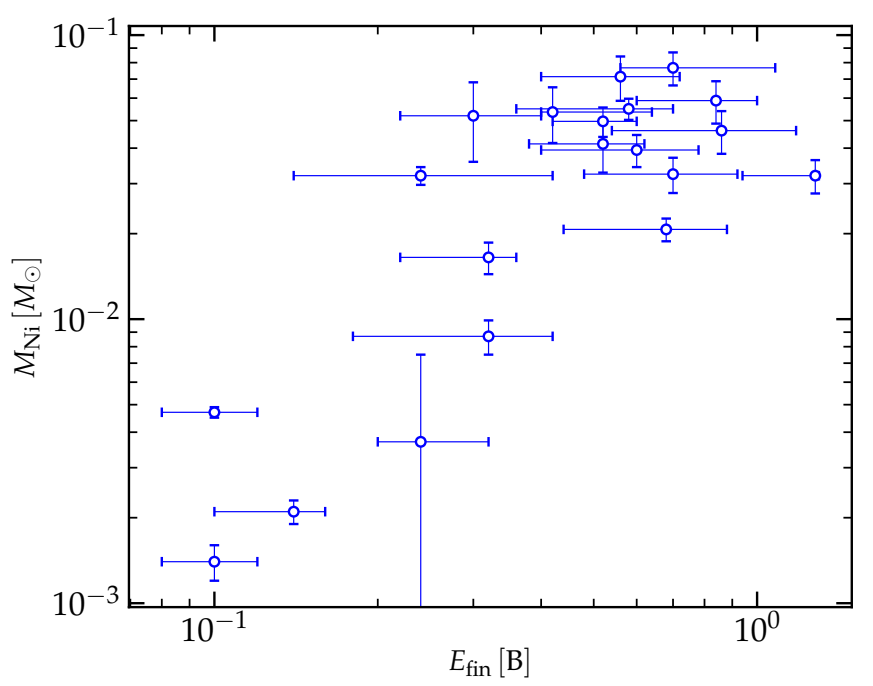

Figure 10. Correlation between the mass of ${ }^{56} \mathrm{Ni}$ and the explosion energy, obtained from our numerical fits.

masses of the progenitors and the explosion energies. The arrows indicate events that only have an upper limit on the CSM mass. From the top panel, there does not appear to be a strong correlation between $M_{\mathrm{CSM}}$ and $M_{\mathrm{ZAMS}}$. Nevertheless, all of the events where we only found CSM mass upper limits are below $M_{\text {ZAMS }} \sim 12 M_{\odot}$, which may be a result of our modeling or due to something physical that should be further explored.

On the other hand, the lower panel of Figure 11 suggests that there is indeed a correlation between $M_{\mathrm{CSM}}$ and the explosion energy. This general correlation was to be expected, since recent studies confirm that SNe IIL have somewhat higher explosion energies per unit ejecta mass than SNe IIP (see, for example, Faran et al. 2014a; Gall et al. 2015) and the steeper decline of SNe IIL is due to a larger amount of dense CSM (Morozova et al. 2017). The important new thing that we find here is that the correlation between $M_{\mathrm{CSM}}$ and explosion energy seems to extend continuously and roughly linearly along the entire sample. What this actually means for the mechanism that generates the dense CSM is unclear. One possibility is that whatever process that generates the CSM also decreases the gravitational binding energy of the star. Then the mechanism that unbinds the star, commonly presumed to be neutrino heating, is more able to create a more energetic explosion.

Figure 12 shows the inferred radii of the CSM versus the ZAMS masses of the progenitors (top panel) and the explosion energies (bottom panel). There appears to be no clear correlation between $R_{\text {ext }}$ and the explosion energy. On the other hands, there may be indication that the dense CSM is more extended for larger $M_{\mathrm{CSM}}$. This radius is related to both the duration and velocity of the CSM. Generally speaking, these values $R_{\text {ext }}$ are only a few times larger than the progenitors' radii. This indicates that no matter the velocity, the dense CSM must have been generated soon before the explosion. For example, assuming the velocity of $10 \mathrm{~km} \mathrm{~s}^{-1}$ gives a timescale of just years (and maybe just months for higher velocities). The advanced stages of nuclear burning could act on such timescales, but the key issue is whether there is a way to harness the energy from this burning to generate the dense CSM. There have been a number of theoretical studies on how to generate pre-explosion outbursts Yoon \& Cantiello (2010); Arnett \& Meakin (2011); Quataert \& Shiode (2012); Shiode
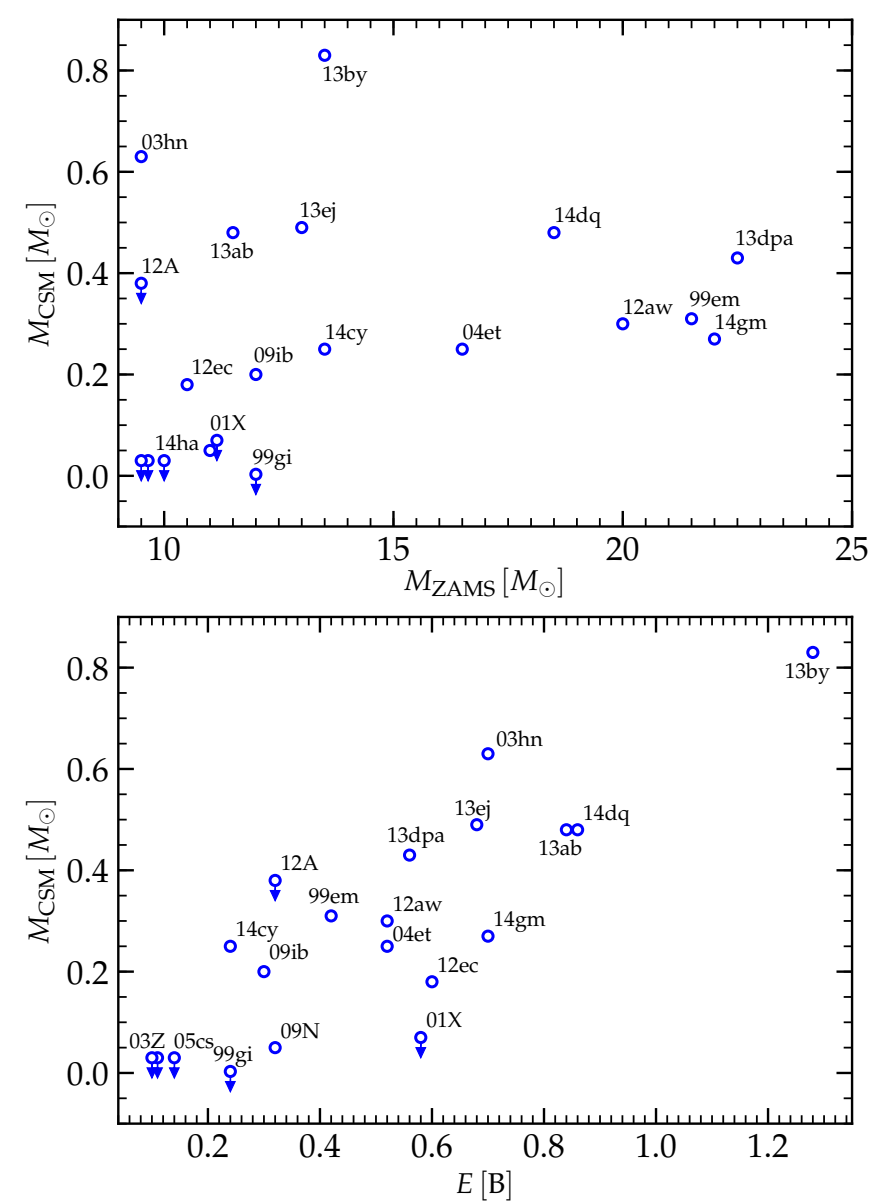

Figure 11. CSM mass versus ZAMS mass (top panel) and explosion energy (bottom panel), obtained from our numerical light curve fits. Arrows indicate $\mathrm{SNe}$ for which we only have upper limits on CSM mass from Table 2.

\& Quataert (2014); Smith \& Arnett (2014); Moriya \& Langer (2015); Woosley \& Heger (2015); Quataert et al. (2016), and most recently one of the most detailed studied of outbursts was modeled by Fuller (2017) using open source stellar evolution code MESA (Paxton et al. 2011, 2013, 2015). Nevertheless, it has yet to be shown that these mechanisms can exactly reproduce the dense CSM properties as we need here.

\subsection{Other implications of dense CSM}

A number of observational works on the early SN II light curves point out that they typically rise faster than the analytical and hydrodynamical light curves obtained from standard RSG models (see, for example, Gall et al. 2015; GonzálezGaitán et al. 2015). As a consequence, fitting the early SN II light curves with the analytical and numerical models resulted in relatively small $\left(\sim 500 R_{\odot}\right)$ estimated radii of their progenitors (Dessart et al. 2013; González-Gaitán et al. 2015; Garnavich et al. 2016; see, however, Shussman et al. 2016; Rubin et al. 2016). These values are on the lower end of the radii estimated from the observations of galactic and Magellanic Clouds RSGs by Levesque et al. (2005) and Levesque et al. (2006), which lie in the range between $\sim 400$ and $\sim 1500 R_{\odot}$. At the same time, a more recent study by Davies et al. (2013) suggests that the previous works could underestimate the RSG temperatures and, consequently, overestimate their radii. For comparison, the radii of the KEPLER models we use vary between $\sim 400$ and $\sim 1400 R_{\odot}$ without the CSM, and similar values for the models with the same ZAMS masses are 

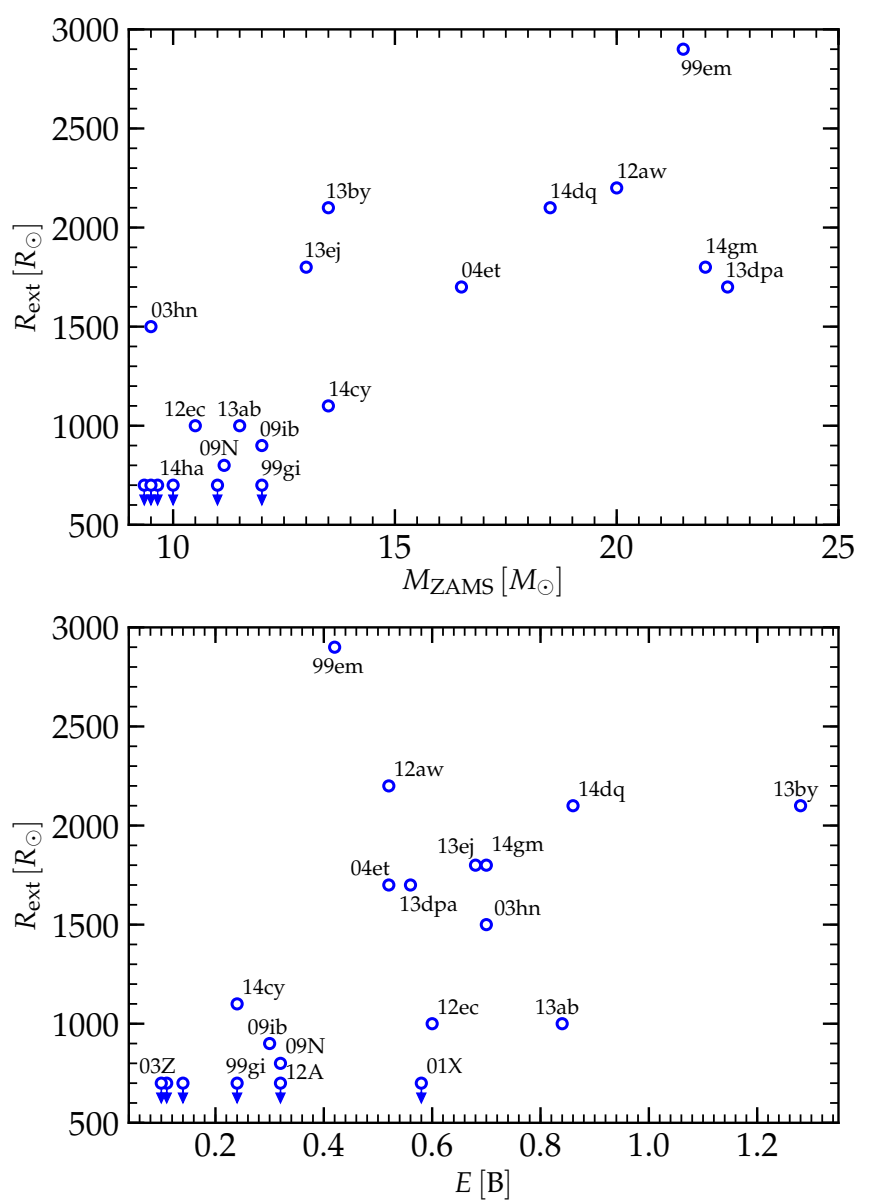

Figure 12. CSM radius versus ZAMS mass (top panel) and explosion energy (bottom panel), obtained from the numerical light curve fits. Arrows indicate SNe for which we only have upper limits on CSM mass from Table 2.

obtained in Renzo et al. (2017). Our current study shows that adding dense CSM to otherwise standard RSGs leads to faster rise times, even though the total radius of the models increases. As can be seen from Figures 2 and 3, this effect is weaker for the ultraviolet bands, which can have the same rise time with and without CSM, but becomes very strong in red bands, where it is crucial for reproducing the early rise and maximum. Therefore, CSM may serve as an explanation of the fast rise of the early SN II light curves rather than the common explanation of changing the radius of the underlying RSGs.

Although we focus on photometric light curves, the presence of a dense CSM around otherwise normal SNe IIP has also been recently suggested by the spectroscopic observations of a number of events (see, for example, Quimby et al. 2007; Kiewe et al. 2012; Yaron et al. 2017). The key is to obtain the spectra early enough, since the characteristic lines typically disappear within a day after the explosion. The material probed by these observations is smaller in mass and larger in radius than what we study here (see discussions in Yaron et al. 2017; Dessart et al. 2017), so the exact relation between these two components of CSM is not clear. It is important to note that in our models the shock breakout happens at the very outer edge of the CSM. This means that at the moment when the first SN light is seen, the bulk of this CSM is optically thick and cannot contribute to the early spectrum. In addition, Dessart et al. (2017) have shown that a sharp transi- tion between the low and high mass loss states assumed in our models cannot reproduce the narrow emission lines observed, for example, in SN 2013fs. In reality, it is likely that there is a smoother transition between the dense CSM we model in this work and the regular low density RSG wind. One interesting possibility to explain it is by employing an accelerating wind that goes from being dense close to the star and less dense as it accelerates further from the star (Moriya et al. 2017). In this case, the shock breaks out inside the wind, which changes the timescale of the breakout from the expected hours to days (Moriya et al. 2011). Such an extended shock breakout was seen, for example, in early observations of a Type II SN PS113arp by Gezari et al. (2015).

\subsection{Limitations of SNEC}

There are a number of approximations and simplifications made in the numerical code we are using, so it is natural to ask how these impact our inferences of dense CSM. Generally speaking, comparison between the light curves generated by SNEC and the multi-group radiation-hydrodynamic code STELLA (Blinnikov \& Bartunov 1993; Baklanov et al. 2005; Blinnikov et al. 2006; Blinnikov \& Bartunov 2011; Kozyreva et al. 2017) that uses the same RSG progenitor shows good agreement in the bolometric luminosity and plateau duration (P. Baklanov, S. Blinnikov, private communication). Nevertheless, one of the largest discrepancies between the color light curves is seen during the early rise, where SNEC light curves rise consistently faster than STELLA light curves, reaching the same magnitudes $\sim 3-5$ days earlier ${ }^{7}$. This can be explained by incomplete thermal equilibrium between the radiation and matter at the photosphere, which is captured by STELLA but not by SNEC (the current version of SNEC uses the assumption of local thermodynamical equilibrium across the entire model). In the more realistic case, the observed temperature is set in a region deeper than the photosphere, where radiation processes are able to couple the gas and radiation (Nakar \& Sari 2010). The value of the observed temperature is therefore higher, and it takes longer for it to drop down to the values corresponding to the black body maxima in the optical bands. This leads to the slower rise in these bands as found by STELLA. As a consequence, we expect that it would be even more challenging for the multigroup radiation-hydrodynamic codes to reproduce the observed SN IIP light curves using stellar evolution RSG models without invoking some sort of CSM. Therefore, the mass of the CSM needed to fit the light curves with these codes will be probably even larger than our estimates. This is roughly consistent with our modeling of the SN 2013fs where the CSM mass is estimated to be $0.47 M_{\odot}$ (Morozova et al. 2017) and the work of Moriya et al. (2017) where it is estimated to be $0.5 M_{\odot}$.

Another limitation is related to the stiff inner boundary in SNEC, which causes numerical problems when we explode high ZAMS mass progenitors with low explosion energies. In Figure 4, the SNe that are most strongly affected by this issue are $1999 \mathrm{em}, 1 \mathrm{sq} 13 \mathrm{dpa}, 2014 \mathrm{cy}$ and asassn14ha. In order to check how this alters the inference of minimum and maximum ZAMS masses of Type II progenitors, we excluded these four

7 The largest disagreement is seen in the $U$ - and $B$-band light curves, which drop faster in STELLA simulations after day $\sim 20$ due to the proper treatment of the iron line opacity. Since we are aware of this problem in our simulations, which is the main reason we do not fit the observed light curves in $U$ - and $B$-bands, we omit the discussion of this effect in the text. 
$\mathrm{SNe}$ from the set and repeated the analysis shown in Figure 9. Without these $\mathrm{SNe}$, the minimum mass $M_{\mathrm{ZAMS}}$, min does not change, while the maximum mass $M_{\mathrm{ZAMS}}$ max changes only slightly from $22.9 M_{\odot}$ to $22.5 M_{\odot}$. Therefore, we believe that the main conclusions of our study do not depend on this problem.

\section{CONCLUSIONS}

Using modeling of the multi-band light curves of twenty $\mathrm{SNe}$ II, we have for the first time constrained the progenitor ZAMS mass, explosion energy, and the mass and radial extent of dense CSM. For $25 \%$ of the SNe we infer ZAMS masses larger than $\sim 17 M_{\odot}$, the maximum limit suggested by the pre-explosion imaging. We show that the mass distribution we infer for our sample is consistent with a Salpeter distribution with a minimum and maximum ZAMS masses for the SNe II progenitors are equal to 10.4 and $22.9 M_{\odot}$, respectively. This is in rough agreement with the observed masses of RSGs and suggests a solution to the RSG problem.

Our results imply that dense CSM is very common amongst SNe IIP, at least $70 \%$ of our fits benefit substantially from including it in the model. The largest amount of CSM (in mass) is expected from IIL-like events, while underluminous $\mathrm{SNe}$ from the low mass progenitors have the smallest amount. The radii of the CSM that we deduce are quite small, in a range of $\sim 800-3000 R_{\odot}$, which argues that whatever mechanism generates the CSM occurs years if not months before the explosion. Given these short timescales, the formation of CSM may be related to the advanced stages of nuclear burning in the stellar interior, which have recently been studied as a mechanism for generating mass outbursts (see Quataert \& Shiode 2012; Shiode \& Quataert 2014; Woosley \& Heger 2015; Quataert et al. 2016; Fuller 2017).

With this sample we are able to explore whether the properties of the dense CSM are related to other aspects of the progenitor or explosion. The main correlations we find are that larger ZAMS masses have CSM at larger radii and larger energy explosions have larger mass CSM. In the former case, this may be related to the time when the dense CSM is generated. In the latter case, there is a suggestion that whatever generates the dense CSM may also make the progenitor star easier to explode. There is of course a many decades long history of trying to understand how core-collapse SNe explode by way of the neutrino mechanism with more failure than success. One possible solution is that whatever mechanism generates the dense CSM may also change the structure of the star in a critical way that could help make the star easier to unbind. Such a hypothesis will hopefully be explored in future theoretical work by utilizing non-standard RSG structures (motivated by the need to generate dense CSM) in the most sophisticated calculations that investigate the neutrino mechanism.

We acknowledge helpful discussions with and feedback from A. Burrows, N. Smith, J. Stone and D. Radice. We thank P. Baklanov, S. Blinnikov, C. Ott, E. Sorokina and T. Moriya for comparison between SNEC and STELLA light curves. Computations were performed on the TIGER cluster at Princeton University. V. M. acknowledges funding support from the Lyman Spitzer Professorship at Princeton University and NSF Grant AST-1714267. A.L.P. acknowledges financial support for this research from a Scialog award made by the
Research Corporation for Science Advancement.

\section{REFERENCES}

Adams, S. M., Kochanek, C. S., Gerke, J. R., Stanek, K. Z., \& Dai, X. 2017, MNRAS, 468, 4968

Anderson, J. P., González-Gaitán, S., Hamuy, M., et al. 2014, ApJ, 786, 67

Arcavi, I., Gal-Yam, A., Cenko, S. B., et al. 2012, ApJ, 756, L30

Arnett, W. D. 1980, ApJ, 237, 541

Arnett, W. D., \& Meakin, C. 2011, ApJ, 733, 78

Baklanov, P. V., Blinnikov, S. I., \& Pavlyuk, N. N. 2005, Astronomy Letters, 31, 429

Barbon, R., Ciatti, F., \& Rosino, L. 1979, A\&A, 72, 287

Bersten, M. C., Benvenuto, O., \& Hamuy, M. 2011, ApJ, 729, 61

Blinnikov, S. I., \& Bartunov, O. S. 1993, A\&A, 273, 106

-. 2011, STELLA: Multi-group Radiation Hydrodynamics Code, Astrophysics Source Code Library

Blinnikov, S. I., Röpke, F. K., Sorokina, E. I., et al. 2006, A\&A, 453, 229

Bose, S., Valenti, S., Misra, K., et al. 2015, MNRAS, 450, 2373

Bruenn, S. W., Lentz, E. J., Hix, W. R., et al. 2016, ApJ, 818, 123

Burrows, A., Vartanyan, D., Dolence, J. C., Skinner, M. A., \& Radice, D. 2016, ArXiv e-prints

Cardelli, J. A., Clayton, G. C., \& Mathis, J. S. 1989, ApJ, 345, 245

Chakraborti, S., Ray, A., Smith, R., et al. 2016, ApJ, 817, 22

Chevalier, R. A., \& Irwin, C. M. 2011, ApJ, 729, L6

Chugai, N. N. 1991, Soviet Astronomy Letters, 17, 210

Chugai, N. N., Chevalier, R. A., \& Utrobin, V. P. 2007, ApJ, 662, 1136

Clausen, D., Piro, A. L., \& Ott, C. D. 2015, ApJ, 799, 190

Crowther, P. A. 2007, ARA\&A, 45, 177

Dall'Ora, M., Botticella, M. T., Pumo, M. L., et al. 2014, ApJ, 787, 139

Davies, B., \& Beasor, E. R. 2018, MNRAS, 474, 2116

Davies, B., Kudritzki, R.-P., Plez, B., et al. 2013, ApJ, 767, 3

Dessart, L., Hillier, D. J., \& Audit, E. 2017, ArXiv e-prints

Dessart, L., Hillier, D. J., Waldman, R., \& Livne, E. 2013, MNRAS, 433, 1745

Dhungana, G., Kehoe, R., Vinko, J., et al. 2016, ApJ, 822, 6

Dwarkadas, V. V. 2014, MNRAS, 440, 1917

Faran, T., Poznanski, D., Filippenko, A. V., et al. 2014a, MNRAS, 445, 554 -. 2014b, MNRAS, 442, 844

Fraser, M. 2016, MNRAS, 456, L16

Fraser, M., Maund, J. R., Smartt, S. J., et al. 2014, MNRAS, 439, L56

Fuller, J. 2017, ArXiv e-prints

Gall, E. E. E., Polshaw, J., Kotak, R., et al. 2015, A\&A, 582, A3

Garnavich, P. M., Tucker, B. E., Rest, A., et al. 2016, ApJ, 820, 23

Gezari, S., Jones, D. O., Sanders, N. E., et al. 2015, ApJ, 804, 28

González-Gaitán, S., Tominaga, N., Molina, J., et al. 2015, MNRAS, 451, 2212

Groh, J. H., Meynet, G., Georgy, C., \& Ekström, S. 2013, A\&A, 558, A131

Hamuy, M. 2003, ApJ, 582, 905

Huang, F., Wang, X., Zhang, J., et al. 2015, ApJ, 807, 59

Jerkstrand, A., Fransson, C., Maguire, K., et al. 2012, A\&A, 546, A28

Jerkstrand, A., Smartt, S. J., Fraser, M., et al. 2014, MNRAS, 439, 3694

Kasen, D., \& Woosley, S. E. 2009, ApJ, 703, 2205

Kiewe, M., Gal-Yam, A., Arcavi, I., et al. 2012, ApJ, 744, 10

Kochanek, C. S. 2014, ApJ, 785, 28

Kochanek, C. S., Khan, R., \& Dai, X. 2012, ApJ, 759, 20

Kozyreva, A., Gilmer, M., Hirschi, R., et al. 2017, MNRAS, 464, 2854

Levesque, E. M., Massey, P., Olsen, K. A. G., et al. 2005, ApJ, 628, 973

-. 2006, ApJ, 645, 1102

Li, W., Van Dyk, S. D., Filippenko, A. V., et al. 2006, ApJ, 641, 1060

Lovegrove, E., \& Woosley, S. E. 2013, ApJ, 769, 109

Lovegrove, E., Woosley, S. E., \& Zhang, W. 2017, ArXiv e-prints

Maguire, K., Di Carlo, E., Smartt, S. J., et al. 2010, MNRAS, 404, 981

Maguire, K., Jerkstrand, A., Smartt, S. J., et al. 2012, MNRAS, 420, 3451

Massey, P., DeGioia-Eastwood, K., \& Waterhouse, E. 2001, AJ, 121, 1050

Mauerhan, J. C., Van Dyk, S. D., Johansson, J., et al. 2017, ApJ, 834, 118

Maund, J. R. 2017, MNRAS, 469, 2202

Maund, J. R., Reilly, E., \& Mattila, S. 2014, MNRAS, 438, 938

Maund, J. R., Fraser, M., Smartt, S. J., et al. 2013, MNRAS, 431, L102

Misra, K., Pooley, D., Chandra, P., et al. 2007, MNRAS, 381, 280

Moriya, T., Tominaga, N., Blinnikov, S. I., Baklanov, P. V., \& Sorokina, E. I. 2011, MNRAS, 415, 199

Moriya, T. J., \& Langer, N. 2015, A\&A, 573, A18

Moriya, T. J., Yoon, S.-C., Gräfener, G., \& Blinnikov, S. I. 2017, ArXiv e-prints

Morozova, V., Piro, A. L., Renzo, M., \& Ott, C. D. 2016, ApJ, 829, 109

Morozova, V., Piro, A. L., Renzo, M., et al. 2015, ApJ, 814, 63

Morozova, V., Piro, A. L., \& Valenti, S. 2017, ApJ, 838, 28

Müller, B., Janka, H.-T., \& Marek, A. 2012, ApJ, 756, 84

Nadezhin, D. K. 1980, Ap\&SS, 69, 115

Nadyozhin, D. K. 2003, MNRAS, 346, 97

Nagy, A. P., \& Vinkó, J. 2016, A\&A, 589, A53

Nakar, E., Poznanski, D., \& Katz, B. 2016, ApJ, 823, 127

Nakar, E., \& Sari, R. 2010, ApJ, 725, 904

Ofek, E. O. 2014, MATLAB package for astronomy and astrophysics, Astrophysics Source Code Library

Ofek, E. O., Rabinak, I., Neill, J. D., et al. 2010, ApJ, 724, 1396 
Özel, F., Psaltis, D., Narayan, R., \& McClintock, J. E. 2010, ApJ, 725, 1918

Özel, F., Psaltis, D., Narayan, R., \& Santos Villarreal, A. 2012, ApJ, 757, 55

Paczyński, B. 1983, ApJ, 267, 315

Paxton, B., Bildsten, L., Dotter, A., et al. 2011, ApJS, 192, 3

Paxton, B., Cantiello, M., Arras, P., et al. 2013, ApJS, 208, 4

Paxton, B., Marchant, P., Schwab, J., et al. 2015, ApJS, 220, 15

Pejcha, O., \& Thompson, T. A. 2015, ApJ, 801, 90

Piro, A. L. 2013, ApJ, 768, L14

Piro, A. L., Muhleisen, M., Arcavi, I., et al. 2017, ApJ, 846, 94

Popov, D. V. 1993, ApJ, 414, 712

Poznanski, D. 2013, MNRAS, 436, 3224

Pumo, M. L., Zampieri, L., Spiro, S., et al. 2017, MNRAS, 464, 3013

Quataert, E., Fernández, R., Kasen, D., Klion, H., \& Paxton, B. 2016,

MNRAS, 458, 1214

Quataert, E., \& Shiode, J. 2012, MNRAS, 423, L92

Quimby, R. M., Wheeler, J. C., Höflich, P., et al. 2007, ApJ, 666, 1093

Radice, D., Burrows, A., Vartanyan, D., Skinner, M. A., \& Dolence, J. C. 2017, ArXiv e-prints

Renzo, M., Ott, C. D., Shore, S. N., \& de Mink, S. E. 2017, ArXiv e-prints

Rubin, A., \& Gal-Yam, A. 2016, ArXiv e-prints

Rubin, A., Gal-Yam, A., De Cia, A., et al. 2016, ApJ, 820, 33

Sanders, N. E., Soderberg, A. M., Gezari, S., et al. 2015, ApJ, 799, 208

Shiode, J. H., \& Quataert, E. 2014, ApJ, 780, 96

Shussman, T., Waldman, R., \& Nakar, E. 2016, ArXiv e-prints

Smartt, S. J. 2009, ARA\&A, 47, 63

Smartt, S. J., Eldridge, J. J., Crockett, R. M., \& Maund, J. R. 2009, MNRAS, 395, 1409

Smith, N., \& Arnett, W. D. 2014, ApJ, 785, 82

Smith, N., Li, W., Filippenko, A. V., \& Chornock, R. 2011, MNRAS, 412, 1522

Spiro, S., Pastorello, A., Pumo, M. L., et al. 2014, MNRAS, 439, 2873
Sukhbold, T., Ertl, T., Woosley, S. E., Brown, J. M., \& Janka, H.-T. 2016, ApJ, 821, 38

Sukhbold, T., \& Woosley, S. E. 2014, ApJ, 783, 10

Summa, A., Hanke, F., Janka, H.-T., et al. 2016, ApJ, 825, 6

Suwa, Y., Yamada, S., Takiwaki, T., \& Kotake, K. 2016, ApJ, 816, 43

Swartz, D. A., Wheeler, J. C., \& Harkness, R. P. 1991, ApJ, 374, 266

Takáts, K., Pumo, M. L., Elias-Rosa, N., et al. 2014, MNRAS, 438, 368

Takáts, K., Pignata, G., Pumo, M. L., et al. 2015, MNRAS, 450, 3137

Tomasella, L., Cappellaro, E., Fraser, M., et al. 2013, MNRAS, 434, 1636

Utrobin, V. P. 2007, A\&A, 461, 233

Utrobin, V. P., \& Chugai, N. N. 2008, A\&A, 491, 507

-. 2009, A\&A, 506, 829

-. 2013, A\&A, 555, A145

Utrobin, V. P., Wongwathanarat, A., Janka, H.-T., \& Mueller, E. 2017, ArXiv e-prints

Valenti, S., Sand, D., Stritzinger, M., et al. 2015, MNRAS, 448, 2608

Valenti, S., Howell, D. A., Stritzinger, M. D., et al. 2016, MNRAS, 459, 3939

Van Dyk, S. D., Cenko, S. B., Poznanski, D., et al. 2012, ApJ, 756, 131

Walmswell, J. J., \& Eldridge, J. J. 2012, MNRAS, 419, 2054

Weaver, T. A., Zimmerman, G. B., \& Woosley, S. E. 1978, ApJ, 225, 1021

Woosley, S. E., \& Heger, A. 2007, Phys. Rep., 442, 269

-. 2015, ApJ, 810, 34

Yaron, O., Perley, D. A., Gal-Yam, A., et al. 2017, Nature Physics, 13, 510

Yoon, S.-C., \& Cantiello, M. 2010, ApJ, 717, L62

Yuan, F., Jerkstrand, A., Valenti, S., et al. 2016, MNRAS, 461, 2003

Zaghloul, M. R., Bourham, M. A., \& Doster, J. M. 2000, J. Phys. D Appl.

Phys., 33, 977 